\title{
On Two-Dimensional Supergravity and the Quantum Super-Möbius Group
}

\author{
Jean-Loup Gervais and Bruno Rostand \\ Laboratoire de Physique Théorique de l'École Normale Supérieure`, 24, rue Lhomond, \\ F-75231 Paris Cedex 05, France
}

Received March 15, 1991

\begin{abstract}
We describe the quantum-group structure of two-dimensional $N=1$ supergravity in the conformal gauge. The operator-algebra of this (superLiouville) theory is shown to correspond to a quantum deformation of the superMöbius group, which provides a new solution of Yang and Baxter's equation. This structure is used to investigate the strong-coupling regime of the theory $(1 \leqq d \leqq 9)$. For $d=5$, a unitary truncation theorem - similar to the one of the bosonic case - is derived for the fundamental representation.
\end{abstract}

\section{Introduction}

In recent articles [1-5], the algebraic connection between two-dimensional gravity and the quantum group $U_{q}(s l(2))$ has been unravelled. The intimate connection between the former and the latter has led to significant progress, and, in particular, has allowed one of us to derive the unitary truncation theorem $[4,5]$ for the strongcoupling regime, thereby proving a long-standing conjecture $[6,3]$, and showing that strongly coupled $2 D$ gravity makes sense for central charges $C=7,13$, and 19 . The extension of this discussion to bosonic Toda theories (corresponding probably to $W$-gravity in the "conformal gauge") has led [7] to new quantum deformations of the Lie algebras $A_{N}$ for $N>1$, that describe their underlying algebraic structures. Partial evidence for the existence of a unitary decoupling theorem, similar to the one of $[4,5]$ have been obtained [3] for the $A_{2}$ Toda theory. So far, however, these recent algebraic techniques have not yet been applied to supergravity in the conformal gauge, that is to the $N=1$ super-Liouville theory, which has been extensively studied [8-10] in the early period after Polyakov's work on the Weyl anomaly [11], using the methods initially developed for the Liouville theory $[12-15,6,16,17]$. More recently striking properties have been

* Unité Propre du Centre National de la Recherche Scientifique, associée à l'École Normale Supérieure et à l'Université de Paris-Sud 
derived for the associated non-critical superstrings [18-20], assuming the existence of a unitary decoupling theorem similar to the one of $[2,4]$. Moreover, the recent developments of matrix models do not seem to apply straightforwardly to the case of supergravity which must be studied in the continuum. It is thus interesting to apply the quantum-group techniques of [1-5] to this problem, and this is the aim of the present article.

In Sect. 2, we recall the basic operator-algebra in the Bloch-wave basis where braiding matrices and fusion coefficients depend upon the quasi-momentum (eigenvalue of the monodromy matrix). In Sect. 3, a new basis of fields is introduced so that this dependence disappears, and a quantum-group structure of the usual type comes out. This allows us to derive the corresponding quantum deformation of the super-Möbius group, its universal $R$ matrix, and co-products. One arrives at a new solution of Yang and Baxter's equation, whose differences with the one previously associated $[21,22]$ with quantum $\operatorname{osp}(1,2)$, are spelled out. In the next section, this structure is used to investigate the unitary decoupling for central charge ${ }^{1} C=5$. The decoupling is proven for the fundamental family of fields, which generates the full operator algebra by fusion. Concluding remarks are made in Sect. 5. Some background-material is recalled in Appendix A, while Appendix B contains technical details for the change of basis discussed in Sect. 3.

\section{The Basic Exchange Algebra for the Chiral Fields}

Our starting point will be the exchange algebra derived in [10] for the superfields that appear in the chiral decomposition of the super-metric and are eigenstates of the monodromy matrix (Bloch waves). Some details are recalled in Appendix A where our notations are spelled out. At the present stage of the discussion, each chirality is studied separately, and we consider only one of them, explicitly, since they are similar (up to subtleties that are discussed in [23] for the bosonic case). The basic objects are super-fields $\psi_{j}(\sigma, \theta), j=1,2$, and 3 ; where $0 \leqq \sigma \leqq 2 \pi$ is a coordinate on the unit circle, and $\theta$ is a fermionic variable such that $\theta^{2}=0$. As recalled in Appendix A, the fields $\psi_{j}$ may be expressed in terms of one bosonic and one fermionic free fields. The latter may be chosen as periodic on the unit circle (in the Ramond sector), or antiperiodic (in the Neveu-Schwartz sector). The fields $\psi_{j}$ are periodic up to a multiplicative constant:

$$
\begin{aligned}
& \psi_{1}(\sigma+2 \pi, \theta)=e^{i h} e^{-2 i h \varpi} \psi_{1}(\sigma, \varsigma \theta), \\
& \psi_{2}(\sigma+2 \pi, \theta)=\varsigma \psi_{2}(\sigma, \varsigma \theta), \\
& \psi_{3}(\sigma+2 \pi, \theta)=e^{i h} e^{2 i h \varpi} \psi_{3}(\sigma, \varsigma \theta) .
\end{aligned}
$$

The parameter $\zeta$ is equal to 1 , in the Ramond (R) sector, or -1 in the NeveuSchwarz (NS) sector. We shall most of the time work in the Neveu-Schwarz sector. The modifications needed to deal with the Ramond sector will be indicated at suitable places. $h$ is an arbitrary parameter related to the coupling constant. It will be shown to be the quantum-deformation-parameter of the super-Möbius group.

\footnotetext{
1 This definition of central charge is usually denoted by $\hat{C}$, but in this article hats have a different
} meaning so that we use $C$ instead 
The central charge of the super-Virasoro algebra is given by

$$
C=1+2\left(\sqrt{\frac{h}{\pi}}+\sqrt{\frac{\pi}{h}}\right)^{2} .
$$

$\varpi$ is a quasi-momentum operator which is shifted by the $\psi$ fields. Many operators we shall encounter are functions of $\varpi$. It will be important to keep in mind that for any function $f$, one has

$$
\psi_{j}(\sigma, \theta) f(\varpi)=f\left(\varpi+\varepsilon_{j}\right) \psi_{j}(\sigma, \theta)
$$

where

$$
\varepsilon_{3}=-\varepsilon_{1}=1 \text {, and } \varepsilon_{2}=0 \text {. }
$$

Some details about the origin of these operators in the super-Liouville theory are given in Appendix A, following [8-10], for completeness. They are not really needed to understand the forthcoming discussion, if one takes for granted the existence of the exchange algebra, to be recalled next. For definiteness, we restrict ourselves to the half circle $0 \leqq \sigma \leqq \pi$, where the $\psi$-fields obey the exchange algebra

$$
\psi_{j}(\sigma, \theta) \psi_{k}\left(\sigma^{\prime}, \theta^{\prime}\right)=\sum_{l, m=1,2,3} S_{j k}^{l m}\left(\sigma-\sigma^{\prime}, \varpi\right) \psi_{l}\left(\sigma^{\prime}, \theta^{\prime}\right) \psi_{n}(\sigma, \theta)
$$

where, in the Neveu-Schwarz sector

$$
\begin{aligned}
S_{13}^{13}(x, \varpi)= & S_{31}^{31}(x,-\varpi)=\frac{\sin (h) \cos (h / 2)}{\cos [h(\varpi-1 / 2)] \sin [h \varpi]} e^{-i h(2 \varpi-1) \varepsilon(x)}, \\
S_{13}^{31}(x, \varpi)= & S_{31}^{13}(x,-\varpi)=\frac{\sin [h(\varpi+1)] \cos [h(\varpi+1 / 2)]}{\sin [h \varpi] \cos [h(\varpi-1 / 2)]} e^{i h \varepsilon(x)}, \\
S_{13}^{22}(x, \varpi)= & S_{31}^{22}(x,-\varpi)=i \varepsilon(x) \frac{\cos (h / 2) \cos [h(\varpi+1 / 2)]}{\sin (h \varpi) \cos [h(\varpi-1 / 2)]} e^{-i h(\varpi-1 / 2) \varepsilon(x)}, \\
S_{22}^{13}(x, \varpi)= & S_{22}^{31}(x,-\varpi)=-i \varepsilon(x) \frac{2 \sin (h / 2) \sin (h) \sin [h(\varpi-1)]}{\cos [h(\varpi-1 / 2)] \cos [h(\varpi+1 / 2)]} \\
& \times e^{-i h(\varpi-1 / 2) \varepsilon(x)}, \quad \\
S_{22}^{22}(x, \varpi)= & -\left(1+\frac{\sin ^{2} h}{\cos [h(\varpi+1 / 2)] \cos [h(\varpi-1 / 2)]}\right),
\end{aligned}
$$

and

$$
\begin{aligned}
& S_{12}^{12}(x, \varpi)=S_{32}^{32}(x,-\varpi)=-i \varepsilon(x) \frac{\sin (h)}{\cos [h(\varpi-1 / 2)]} e^{-i h(\varpi-1 / 2) \varepsilon(x)} \\
& S_{12}^{21}(x, \varpi)=S_{32}^{23}(x,-\varpi)=\frac{\cos [h(\varpi+1 / 2)]}{\cos [h(\varpi-1 / 2)]} \\
& S_{21}^{21}(x, \varpi)=S_{23}^{23}(x,-\varpi)=-i \varepsilon(x) \frac{\sin (h)}{\cos [h(\varpi-1 / 2)]} e^{i h(\varpi-1 / 2) \varepsilon(x)}, \\
& S_{21}^{12}(x, \varpi)=S_{23}^{32}(x,-\varpi)=\frac{\cos [h(\varpi-3 / 2)]}{\cos [h(\varpi-1 / 2)]}
\end{aligned}
$$


and where $\varepsilon(x)$ is the sign of $x$. In the Ramond sector, the exchange matrix is given by the same expressions, with the substitution $\varpi \rightarrow(\varpi+\pi / 2 h)$. Therefore, all the manipulations which we shall describe in the following sections are valid in both sectors. For simplicity we shall describe them explicitly in the Neveu-Schwarz sector only, but one may find their Ramond sector analogs by making the above substitution in all the functions of $\varpi$ involved.

The algebra $(2.6,2.7)$ was derived in $[10]$ from the operator super-Schrödinger equation, which is the quantum version of Eq. (A.12), satisfied by the $\psi$-fields. The normalisation of the operators was left arbitrary. We have specified it so as to simplify the above braiding matrix as much as possible. Some details are spelled out in Appendix B. This structure is directly applicable to the physics of twodimensional super-gravity if $h$ is real (more about the case of complex $h$ below). Then the spectrum of eigenvalues of $\varpi$ is real, in agreement with the fact that the $\psi$ fields shift $\varpi$ by real numbers, as shown by Eqs. $(2.3,2.4)$. In this case, $\varpi$ is a hermitian operator. One may verify from their expressions [10] that the fields $\psi$ then satisfy the hermiticity conditions

$$
\psi_{1}^{\dagger}=\psi_{3}, \quad \psi_{2}^{\dagger}=\psi_{2} .
$$

One may directly verify that this conjugation is consistent with the present operator algebra. First it agrees with Eq. (2.1) if one takes account of the fact that $\varpi$ is an operator that does not commute with the $\psi$ fields. For instance, taking the hermitian conjugate of the first line of (2.1) gives $\psi_{1}^{\dagger}(\sigma+2 \pi, \theta)=e^{-i h} \psi_{1}^{\dagger}(\sigma, \varsigma \theta) e^{2 i h w}$. According to (2.3), this is equivalent to $\psi_{1}^{\dagger}(\sigma+2 \pi, \theta)=e^{i h} e^{2 i h \omega} \psi_{1}^{\dagger}(\sigma, \varsigma \theta)$; so that $\psi_{1}^{\dagger}$ and $\psi_{3}$ have the same monodromy properties, in agreement with (2.8). Second, taking the hermitian conjugate of Eq. (2.5), one obtains

$$
\psi_{k}^{\dagger}\left(\sigma^{\prime}, \theta^{\prime}\right) \psi_{j}^{\dagger}(\sigma, \theta)=\sum_{l, m=1,2,3} \psi_{m}^{\dagger}(\sigma, \theta) \psi_{l}^{\dagger}\left(\sigma^{\prime}, \theta^{\prime}\right)\left(S_{j k}^{l m}\left(\sigma-\sigma^{\prime}, \varpi\right)\right)^{*}
$$

so that, according to Eqs. (2.3), and (2.8), one should have

$$
\left(S_{j k}^{l m}\left(\sigma-\sigma^{\prime}, \varpi-\varepsilon_{l}-\varepsilon_{m}\right)\right)^{*}=S_{k^{\prime} j^{\prime}}^{m^{\prime} l^{\prime}}\left(\sigma^{\prime}-\sigma, \varpi\right)
$$

where indices with primes are such that $1^{\prime}=3,3^{\prime}=1,2^{\prime}=2$. It is straightforward to verify that the expressions (2.6) and (2.7) satisfy this relation for $h$ real and $\varpi$ hermitian. One thus sees that (2.8) is indeed an antilinear automorphism of the operator-algebra.

Before leaving the preliminaries, an important point should be stressed. Solutions of the Yang-Baxter equations associated with super-braiding matrices have already been extensively discussed in the literature $[24,21,25,22]$ introducing matrices with a non-trivial grading. This is, in particular, systematically discussed in [24]. Our starting point is the explicit realisation just recalled of an exchange algebra by super-operators, where the elements of the exchange matrix $S$ do not depend upon the $\theta$ variables, and are thus of a bosonic nature. It follows that contrary to the common practice for the superalgebra, no grading is assumed for any matrix we shall write later on. Tensor products are always assumed to commute. This is consistent with the Yang-Baxter equation which comes out from the associativity of the products of $\psi$ operators:

$$
\begin{aligned}
& \sum_{\varrho, \lambda, \mu} S_{p \lambda}^{l \varrho}\left(\omega, \sigma_{1}-\sigma_{2}\right) S_{j k}^{\lambda \mu}\left(\omega+\varepsilon_{p}, \sigma_{2}-\sigma_{3}\right) S_{\varrho \mu}^{m n}\left(\omega+\varepsilon_{l}, \sigma_{1}-\sigma_{2}\right) \\
& \quad=\sum_{\varrho, \lambda, \mu} S_{p j}^{\mu \lambda}\left(\omega, \sigma_{1}-\sigma_{2}\right) S_{\lambda k}^{\varrho n}\left(\omega+\varepsilon_{\mu}, \sigma_{1}-\sigma_{3}\right) S_{\mu \varrho}^{l n}\left(\omega, \sigma_{2}-\sigma_{3}\right),
\end{aligned}
$$


where the summations run over 1, 2, and 3, and the shifts $\varepsilon_{j}$ are given by Eq. (2.4). These equations have exactly the same form as for the Liouville theory which is purely bosonic [15], and associated $[1,2,4,5]$ with $U_{q}(\operatorname{sl}(2))$. They are thus nongraded Yang-Baxter equations, in agreement with the general philosophy followed in this paper. One will see that $q$-deformations of a super-Lie-algebra nevertheless come out, since the co-product will enjoy unusual properties.

As is always the case, with operators that are periodic up to a phase, the exchange matrix is an explicit function of the quasi-momentum $\varpi$ which is an operator that does not commute with the $\psi$ fields. Thus the Yang-Baxter equation does not take the form which is usual for quantum groups. This question has already been extensively discussed in the bosonic case $[1,2,4,5,3]$.

\section{Connection with the Quantum Super-Möbius Group}

In the previous section, we have recalled the exchange properties of the superVirasoro primary fields which generate the operator-algebra of two-dimensional supergravity. The corresponding braiding matrix has a non-trivial dependence on the quasi-momentum $\varpi$, which implies in particular that it does not commute with the fields. In this section, we shall describe a new basis of fields, whose braiding is independent of $\varpi$; moreover the new braiding matrix so defined will turn out to correspond to a quantum deformation of the super-Möbius algebra, previously exhibited in $[21,22]$. The new fields $\xi_{\alpha}$ are linear combinations of the fields $\psi_{j}$ which we have discussed above, with $\varpi$-dependent coefficients:

$$
\xi_{\alpha}=\sum_{j=1}^{3} u_{\alpha}^{j}(\varpi) \psi_{j}, \quad \alpha=1,2,3 .
$$

The exchange properties of these new fields are described by a matrix $\varrho$ such that

$$
\xi_{\alpha}(\sigma, \theta) \xi_{\beta}\left(\sigma^{\prime}, \theta^{\prime}\right)=\varrho_{\alpha \beta}^{\gamma \delta} \xi_{\gamma}\left(\sigma^{\prime}, \theta^{\prime}\right) \xi_{\delta}(\sigma, \theta) .
$$

Thus the quantities $u_{\alpha}^{j}(\varpi)$ must be such that

$$
\sum_{\gamma, \delta} \varrho_{\alpha \beta}^{\gamma \delta}\left(\sigma-\sigma^{\prime}\right) u_{\gamma}^{i}(\varpi) u_{\delta}^{k}\left(\varpi+\varepsilon_{j}\right)=\sum_{l, m} S_{l m}^{j k}\left(\sigma-\sigma^{\prime}, \varpi\right) u_{\alpha}^{l}(\varpi) u_{\beta}^{m}\left(\varpi+\varepsilon_{l}\right),
$$

where $\varrho_{\alpha \beta}^{\nu \delta}$ may depend on $\left(\sigma-\sigma^{\prime}\right)$, but not on the quasi-momentum $\varpi$. In $[2,7]$ a systematic method for deriving similar changes of field-basis was described in the context of two-dimensional gravity and Toda conformal field theories, respectively. In the present case, the use of this method provides only a partial answer to the question. As explained in detail in Appendix B, the derivation of a $\varpi$-independent braiding matrix requires some extra work; the final answer turns out to be:

$$
\left\{\begin{array}{l}
u_{1}^{j}(\varpi)=(-1)^{\varepsilon_{j}} \exp \left[-i h\left(\varepsilon_{j} \varpi+\frac{\left(\varepsilon_{j}\right)^{2}}{2}\right)\right] \\
u_{3}^{j}(\varpi)=\exp \left[i h\left(\varepsilon_{j} \varpi+\frac{\left(\varepsilon_{j}\right)^{2}}{2}\right)\right],
\end{array}\right.
$$

and

$$
\left\{\begin{array}{l}
u_{2}^{1}(\varpi)=u_{2}^{3}(\varpi)=i a(\varpi) \sqrt{2 \sin (h / 2)} \\
u_{2}^{2}(\varpi)=a(\varpi) \cos (h \varpi) \sqrt{2 / \sin (h / 2)},
\end{array}\right.
$$


where $a(\varpi)$ is a "cocycle" such that

$$
a(\varpi)^{2}=1 \quad \text { and } \quad a(\varpi+1) / a(\varpi)=-1 .
$$

For $\varepsilon\left(\sigma-\sigma^{\prime}\right)=1$ one finds

$$
\begin{aligned}
& \varrho_{11}^{11}=\varrho_{33}^{33}=e^{-i h} ; \quad \varrho_{13}^{31}=\varrho_{31}^{13}=e^{i h} ; \\
& \varrho_{31}^{31}=-\left(1+e^{i h}\right)\left(e^{i h}-e^{-i h}\right) ; \\
& \varrho_{22}^{22}=-1 ; \quad \varrho_{22}^{31}=\varrho_{31}^{22}=i e^{i h / 2}\left(e^{i h}-e^{-i h}\right) ; \\
& \varrho_{12}^{21}=\varrho_{21}^{12}=\varrho_{23}^{32}=\varrho_{32}^{23}=-1 ; \quad \varrho_{21}^{21}=\varrho_{32}^{32}=-\left(e^{i h}-e^{-i h}\right)
\end{aligned}
$$

and the other elements vanish. In matrix form the result is

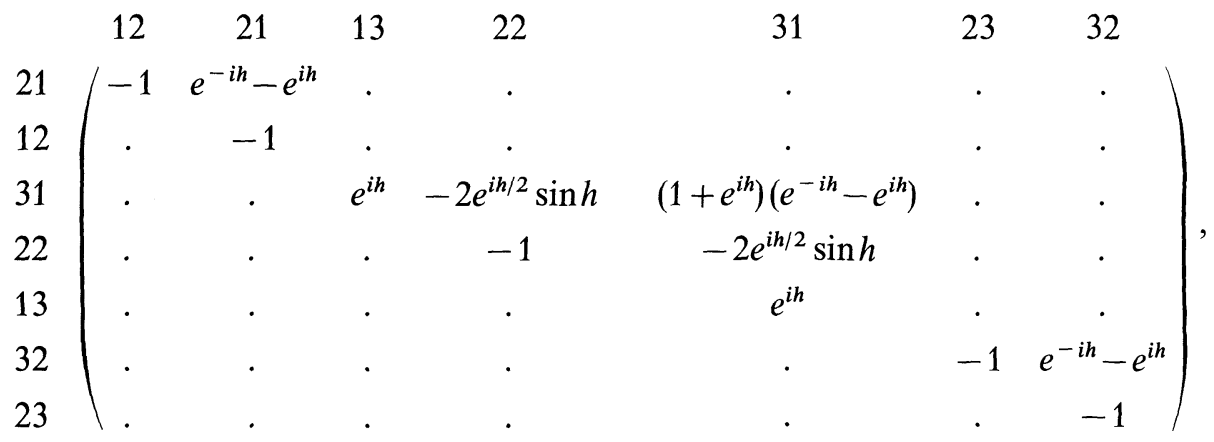

where we have omitted the trivial subspace $(11,33)$. All dependence on the quasimomentum has been eliminated, and the above braiding matrix satisfies the YangBaxter equation without the $\varpi$ dependence appearing in (2.11). Moreover, this also implies that the value of the braiding matrix $\varrho$ in the Ramond sector is identical to that in the Neveu-Schwarz sector, since the two sectors correspond to each other by a shift of $\varpi$.

Before going further in our discussion, let us quote two important properties of the transformation (3.1). First it is such that, for $h$ real and $\varpi$ hermitian, the $\xi$ fields are hermitian. This is easily verified, from Eq. (2.8) by writing

$$
\xi_{\alpha}^{\dagger}=\sum_{j=1}^{3} \psi_{j^{\prime}}\left(u_{\alpha}^{j}(\varpi)\right)^{*}=\sum_{j^{\prime}=1}^{3}\left(u_{\alpha}^{j^{\prime}}\left(\varpi+\varepsilon_{j}\right)\right)^{*} \psi_{j},
$$

and checking that $\left(u_{\alpha}^{j^{\prime}}\left(\varpi+\varepsilon_{j}\right)\right)^{*}=u_{\alpha}^{j}(\varpi)$. This last part is easily verified using (3.4), (3.5), and (3.6), choosing $a(\varpi)$ to be hermitian, as we shall do. It follows that, for the $\xi$ fields, the equivalent of $(2.10)$ reads

$$
\left(\varrho_{\alpha \beta}^{\gamma \delta}\right)^{*}=\bar{\varrho}_{\beta \alpha}^{\gamma \delta}
$$

where $\bar{\varrho}$, which is such that

$$
\sum_{\beta \gamma} \varrho_{\alpha \beta}^{\gamma \delta} \bar{\varrho}_{\gamma \delta}^{\lambda \mu}=\delta_{\alpha, \lambda} \delta_{\beta, \mu}
$$

is the exchange matrix for $\varepsilon=-1$. The other important property concerns the inversion of the transformation (3.1). Following a path [5] similar to the case of 
$U_{q}(\operatorname{sl}(2))$, one verifies that the $u$ 's satisfy pseudo-orthogonality relations of the form

$$
\sum_{\alpha} e^{-i(\alpha-2)(h+\pi) / 2} u_{\alpha}^{j}(\varpi) u_{\alpha^{\prime}}^{k^{\prime}}\left(\varpi+\varepsilon_{k}\right)=\delta_{j, k} C_{j}(\varpi),
$$

where

$$
\left\{\begin{array}{l}
C_{1}(\varpi)=4 \sin (h \varpi) \cos [h(\varpi-1 / 2)] \\
C_{3}(\varpi)=-4 \sin (h \varpi) \cos [h(\varpi+1 / 2)] \\
C_{2}(\varpi)=2 \cos [h(\varpi-1 / 2)] \cos [h(\varpi+1 / 2)] / \sin (h / 2) .
\end{array}\right.
$$

The significance of Eq. (3.12) is that it allows us to invert (3.1) and obtain

$$
\psi_{j}=\sum_{\alpha} \xi_{\alpha} u_{\alpha^{\prime}}^{j^{\prime}}(\varpi) e^{i(\alpha-2)(h+\pi) / 2} / C_{j^{\prime}}(\varpi) .
$$

We now show that the new basis of fields provides a direct relation to a quantum deformation of the super-Möbius group. For a closer correspondence with previous authors $[21,25,22]$, we first redefine the generators of the superMöbius algebra introduced in Appendix A, to map it to the $\operatorname{osp}(1,2)$ algebra:

$$
\left\{\begin{array}{c}
V_{ \pm}=\frac{1}{2} G_{ \pm 1 / 2} \\
J_{ \pm}= \pm L_{ \pm 1} \\
H=-L_{0}
\end{array}\right.
$$

These operators indeed realize the $\operatorname{osp}(1,2)$ algebra:

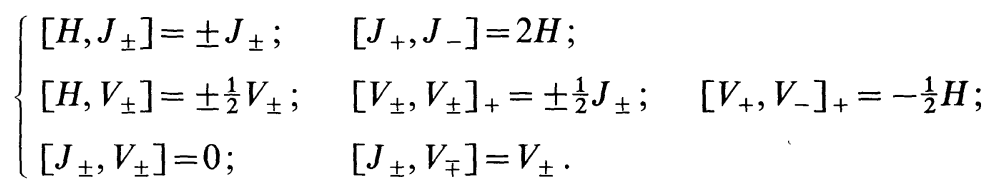

The finite-dimensional representations of this algebra are decomposable in sums of irreducible ones, which are characterized by their integer or half-integer spin $J$, and have dimension $4 J+1$. The fundamental representation $(J=1 / 2)$ has dimension 3 ; we may choose a basis $(|-1 / 2\rangle,|0\rangle,|1 / 2\rangle)$ in which $H$ is diagonal: $H|M\rangle=M|M\rangle$; and

$$
\left\{\begin{array}{l}
V_{+}|-1 / 2\rangle=(1 / 2)|0\rangle \\
V_{+}|0\rangle=(1 / 2)|1 / 2\rangle \\
V_{+}|1 / 2\rangle=0
\end{array}, \quad\left\{\begin{array}{l}
V_{-}|-1 / 2\rangle=0 \\
V_{-}|0\rangle=(1 / 2)|-1 / 2\rangle \\
V_{-}|1 / 2\rangle=-(1 / 2)|0\rangle
\end{array}\right.\right.
$$

The enveloping algebra is generated by $\left(H, V_{ \pm}\right)$, its quantum deformation consists in a modification of the anticommutator of $V_{+}$and $V_{-}$:

$$
\left[V_{+}, V_{-}\right]_{+}=-\frac{1}{4} \frac{\sin (2 h H)}{\sin h}=-\frac{1}{4}[2 H] \text {, }
$$

where the notation \lfloor\rfloor denotes the usual $q$-analog (for $q=e^{i h}$ ),

$$
\lfloor x\rfloor=\frac{\sin (h x)}{\sin h}
$$


and the commutators of $H$ with $V_{ \pm}$are not modified. The comultiplication corresponding to this quantum deformation of the algebra reads ${ }^{2}$

$$
\left\{\begin{array}{l}
\Lambda(H)=1 \otimes H+H \otimes 1 \\
\Lambda\left(V_{ \pm}\right)=e^{i(h \mp \pi) H} \otimes V_{ \pm}+V_{ \pm} \otimes e^{-i(h \mp \pi) H} .
\end{array}\right.
$$

We have derived this result as follows. First the co-product was determined in the spin $1 / 2$ representation by requesting that it commute with the braiding matrix (3.7). Second, assuming that the algebra is undeformed in the spin $1 / 2$ representation, and using the co-product, we derived the deformation (3.18) of the enveloping algebra. The realization of the algebra in the fundamental representation is indeed undeformed because $[2 x]=2 x$ for $x=0, \pm 1 / 2$. From the expression in Eq. (3.17) and the coproduct (3.20), one may obtain the realization of the quantum algebra in higher-dimensional representations. This realization involves a second $q$-analog, defined for integer or half-integer values of $x$ :

$$
\lfloor x\rfloor_{+}=\frac{e^{i h x}-(-1)^{2 x} e^{-i h x}}{e^{i h / 2}+e^{-i h / 2}} .
$$

The eigenvalues of $H$ in the spin $J$ representation are $M=-J$, $-J+1 / 2, \ldots, J-1 / 2, J$; the action of the generators on the states reads

$$
\left\{\begin{aligned}
H|J, M\rangle & =M|J, M\rangle, \\
V_{ \pm}|J, M\rangle & = \pm \frac{1}{2} \sqrt{\lfloor J \mp M\rfloor\lfloor J \pm M+1 / 2\rfloor_{+}}\left|J, M \pm \frac{1}{2}\right\rangle \quad \text { if } J-M \text { is integer } \\
& =\frac{1}{2} \sqrt{\lfloor J \mp M\rfloor_{+}\lfloor J \pm M+1 / 2\rfloor}\left|J, M \pm \frac{1}{2}\right\rangle \text { if } J-M \text { is half-integer. }
\end{aligned}\right.
$$

By exchanging the role of the two vector spaces whose tensor product appears in Eq. (3.13), one obtains a second coproduct:

$$
\left\{\begin{array}{l}
\bar{\Lambda}(H)=1 \otimes H+H \otimes 1 \\
\bar{\Lambda}\left(V_{ \pm}\right)=e^{-i(h \mp \pi) H} \otimes V_{ \pm}+V_{ \pm} \otimes e^{i(h \mp \pi) H} .
\end{array}\right.
$$

Before going on, we note an unusual feature of our co-products: they do not become trivial when $h \rightarrow 0$. As a matter of fact, this is consistent, since we are using ungraded tensor products. The $h=0$ limit of $\Lambda$ and $\bar{\Lambda}$ precisely re-establishes the graduation which is needed for taking tensor products of representations of the undeformed $\operatorname{ssp}(1,2)$ superalgebra. A related point is that, when $h \rightarrow 0$, our braiding matrix tends to the exchange matrix $E_{\alpha \beta}^{\gamma \delta}=\delta_{\alpha, \delta} \delta_{\beta, \gamma}$ multiplied by unexpected minus signs, since $\xi_{1}$ and $\xi_{3}$ become commuting and $\xi_{2}$ anticommuting, but $\xi_{1}$, and $\xi_{3}$ anticommutes with $\xi_{2}$ in this limit. There seems to be no way to re-establish a more standard statistics. This is one of the reasons why we did not introduce any gradation: none seems to be natural. Note that the $\xi$ are super-fields involving a bosonic and a fermionic part to begin with.

We have generalized the braiding matrix (3.7) to all representations by imposing that it relate the two co-products in the usual way. Its expression in terms of the $U_{q}(\operatorname{osp}(1,2))$ generators reads:

$$
\begin{aligned}
R= & -e^{-4 i(h+\pi)(H \otimes H)} \sum_{n \geqq 0}(4 i)^{n}(-1)^{n(n-1) / 2} e^{i n n(n+1) / 4} \frac{\left(e^{i h}-e^{-i h}\right)^{n}}{\lfloor n / 2\rfloor_{+} !} \\
& \left.\times e^{-i n((h-\pi)(H \otimes 1)-(h+\pi)(1 \otimes H))}\left(V_{+}\right)^{n} \otimes\left(V_{-}\right)^{n}\right),
\end{aligned}
$$

2 Notice that, as explained in Sect. 2 our conventions regarding the tensor product of representations of graded algebras differ from those of [24] in that we do not assign a minus sign to the permutation of odd states; more about this below 
where

$$
\lfloor n / 2\rfloor_{+} !=\lfloor n / 2\rfloor_{+}\lfloor(n-1) / 2\rfloor_{+} \ldots\lfloor 1\rfloor_{+}\lfloor 1 / 2\rfloor_{+} \cdot
$$

This $R$-matrix is such that

$$
R \Lambda=\bar{\Lambda} R \text {. }
$$

Its evaluation in the fundamental representation of $O s p(1,2)$ via

$$
\varrho_{M M^{\prime}}^{N^{\prime} N}=\left(\left\langleM\left|\otimes\left\langle M^{\prime}\right|\right) R\left(|N\rangle \otimes\left|N^{\prime}\right\rangle\right)\right.\right.
$$

gives back the braiding matrix of Eq. (3.7), up to the identification $\alpha=2 M+2$. Note that the relation (3.27) between $R$ and $\varrho$ involves a permutation of two indices; thus, as used above, $\varrho$ simply commutes with the co-product $\Lambda$, without the occurrence of $\bar{\Lambda}$ :

$$
\varrho \Lambda=\Lambda \varrho .
$$

Finally, we compare in detail our result with the known expressions for the $q$-deformation of the graded algebra $\operatorname{osp}(1,2)$, which we distinguish by a tilde. We shall use the notations of [22] for definiteness. Our matrice $\varrho$ Eq. (3.7) satisfies the non-graded Yang-Baxter equation. Thus it is analogous to the matrix $\mathscr{\mathscr { R }}$ of [22]. Comparing our co-product with Eq. (10) of [22], one is led to relate the two structures by letting $q$ of [22] equal to $e^{-i(h-\pi)}$. There appears an overall minus sign, which is irrelevant for the Yang-Baxter equations. Calling - $-\tilde{\varrho}$, the braiding matrix so derived, one finds that it is given by

$$
\begin{aligned}
& \tilde{\varrho}_{11}^{11}=\tilde{\varrho}_{33}^{33}=e^{-i h} ; \quad \tilde{\varrho}_{13}^{31}=\tilde{\varrho}_{31}^{13}=e^{i h} ; \\
& \tilde{\varrho}_{31}^{31}=-\left(1-e^{i h}\right)\left(e^{i h}-e^{-i h}\right) ; \\
& \tilde{\varrho}_{22}^{22}=1 ; \quad \tilde{\varrho}_{22}^{31}=-\tilde{\varrho}_{31}^{22}=i e^{i h / 2}\left(e^{i h}-e^{-i h}\right) ; \\
& \tilde{\varrho}_{12}^{21}=\tilde{\varrho}_{21}^{12}=\tilde{\varrho}_{23}^{32}=\tilde{\varrho}_{32}^{23}=-1 ; \quad \tilde{\varrho}_{21}^{21}=\tilde{\varrho}_{32}^{32}=-\left(e^{i h}-e^{-i h}\right) .
\end{aligned}
$$

The differences between (3.7) and (3.29) are only in $\varrho_{31}^{31}, \varrho_{22}^{22}$, and $\varrho_{31}^{22}$, where one sign has changed in each case. One may of course directly verify that both matrices satisfy the Yang-Baxter equations, since the latter leave this sign undetermined. Concerning the co-product, Eq. (3.20) trivially gives

$$
\left(\left\langle\alpha|\otimes\langle\beta|) \Lambda_{ \pm}(|\gamma\rangle \otimes|\delta\rangle)=\delta_{\beta, \delta} f_{\alpha \beta}^{ \pm}\left\langle a\left|V_{ \pm}\right| \gamma\right\rangle+\delta_{\alpha, \gamma} g_{\alpha \beta}^{ \pm}\left\langle\beta\left|\mathbf{V}_{ \pm}\right| \delta\right\rangle\right.\right.
$$

where

$$
f_{\alpha \beta}^{ \pm}=e^{i(h \mp \pi)(\beta-2) / 2}, \quad g_{\alpha \beta}^{ \pm}=e^{-i(h \mp \pi)(\alpha-2) / 2} .
$$

In order to compare with [22], one puts its formula (4), with $q=e^{-i(h-\pi)}$, under the form (3.30). Remembering that it uses graded tensor products, one obtains

$$
\tilde{f}_{\alpha \beta}^{+}=f_{\alpha \beta}^{+}(-1)^{P(\beta)}, \quad \tilde{g}_{\alpha \beta}^{+}=g_{\alpha \beta}^{+} ; \quad \tilde{f}_{\alpha \beta}^{-}=-f_{\alpha \beta}^{-}, \quad \tilde{g}_{\alpha \beta}^{-}=-g_{\alpha \beta}^{+}(-1)^{P(\alpha)},
$$

where $P(\alpha)$ is the graduation-function of [24], which is equal to 1 for $\alpha=2$, and vanishes otherwise. Again there are various sign changes. Although the two sets of formulae are very similar, their quantum-group meaning is quite different, since the matrices $\varrho$ and $\varrho$ do not have the same eigenvalues. This may be checked by comparing their traces $\sum_{\alpha \beta} \varrho_{\alpha \beta}^{\alpha \beta}$ and $\sum_{\alpha \beta} \tilde{\varrho}_{\alpha \beta}^{\alpha \beta}$. 


\section{Strong Coupling Regime with $C=5$}

The previous discussion is directly applicable if $h$ is real. This is the case for the weak-coupling regime of supergravity $(C>9)$, and for super-minimal-models $(C<1)$. In the region $1<C<9, h$ is complex and we have to work more, since the continuation of the formulae just written gives meaningless complex results in general. For this we have to consider the two possible deformation-parameters $h$ and $\hat{h}$ given by Eq. (A.22) together, since they are complex conjugate. In preparing this section, we were aiming at unitary truncations similar to the ones of the bosonic cases [6,3-5], where three equally spaced special values came out. So far, however, we have only been able to treat the case of $C=5$ (analogous to $C=13$ for quantum gravity) only, and this is the subject of the present section. The difficulties with the other choices $(C=3$, and $C=7)$ will be explained at the end.

In general the two possible quantum modifications are related by

$$
h \hat{h}=\pi^{2}, \quad h+\hat{h}=\frac{\pi}{2}(C-5),
$$

so that, in the present case, we have $h+\hat{h}=0$, and $h$ and $\hat{h}$ are pure imaginary. Let us recall that the operators corresponding to $\hat{h}$ are denoted $\hat{\psi}$, and that all quantities involving $\hat{h}$ instead of $h$ are distinguished by a hat. For instance, the exchange properties of the $\hat{\psi}$ fields are given by

$$
\psi_{j}(\sigma, \theta) \hat{\psi}_{k}\left(\sigma^{\prime}, \theta^{\prime}\right)=\sum_{l, m=1,2,3} \hat{S}_{l j k}^{l m}\left(\sigma-\sigma^{\prime}, \hat{\varpi}\right) \hat{\psi}_{l}\left(\sigma^{\prime}, \theta^{\prime}\right) \hat{\psi}_{m}(\sigma, \theta),
$$

where $\hat{S}_{j k}^{l m}$ is deduced from Eqs. $(2.6,2.7)$ by replacing $h$ by $\hat{h}$ and $\varpi$ by $\hat{\varpi}$. We also introduce fields $\hat{\xi}_{\alpha}$ given by the hatted versions of Eqs. $(3.1,3.4,3.5)$. As recalled in Appendix A, the fields are defined in spaces that are direct sums of Verma modules with highest weights of the form

$$
\Delta_{0}\left(\varpi^{(0)}+v+\hat{v} \frac{\pi}{h}\right)=\frac{C-1}{16}\left(\frac{1-\varsigma}{2}\right)-\frac{1}{2}\left\{\left(\varpi^{(0)}+v\right) \sqrt{h / \pi}+\hat{v} \sqrt{\hat{h} / \pi}\right\}^{2}
$$

with $v$ and $\hat{v}$ integers. In the present regime, this formula is complex in general. On the other hand, the representations of the Virasoro algebra in each Verma module are unitary iff $\Delta_{0}$ is real and positive, since the central charge is larger than one. In view of (4.3), this is the case if $v$ and $\hat{v}$ are such that $\left(\varpi^{(0)}+v\right) \sqrt{h / \pi}+\hat{v} \sqrt{h / \pi}$ is pure imaginary. Since $h$ and $\hat{h}$ are complex conjugate, this requires that $v+\hat{v}=v_{0}$, where $v_{0}$ is a constant, and $\left(\varpi^{(0)}+v_{0}\right) \sqrt{h / \pi}$ is pure imaginary. The representation of the Virasoro algebra will be unitary, iff we are able to restrict ourselves consistently to a set of $\varpi$ satisfying these conditions. This restricts the set of operators which can be included in the operator algebra; in particular, $\psi$ or $\hat{\psi}$ fields alone are forbidden to appear. The simplest possible operators are linear combinations of operators of the form

$$
\psi_{13} \sim \psi_{1} \hat{\psi}_{3}, \quad \psi_{31} \sim \psi_{3} \hat{\psi}_{1}, \quad \text { and } \quad \psi_{22} \sim \psi_{2} \hat{\psi}_{2},
$$

where $\sim$ means that one takes the leading order in the operator-product expansion. We take the same normalisation-convention in defining $\psi_{13}, \psi_{31}$, and $\psi_{22}$, so that it becomes irrelevant. These three operators shift the quasi-momentum from $(v, \hat{v})$ to $(v-1, \hat{v}+1),(v+1, \hat{v}-1)$ and $(v, \hat{v})$, respectively.

It will be more powerful to use the $\xi$ fields and introduce

$$
\xi_{13} \sim \xi_{1} \hat{\xi}_{3}, \quad \xi_{31} \sim \xi_{3} \hat{\xi}_{1}, \quad \text { and } \quad \xi_{22} \sim \xi_{2} \hat{\xi}_{2} .
$$


However, we shall start by using $\psi_{j k}$ fields as a warming up. They are not so suitable to carry out the complete proof, but are simpler to begin with. The ansatz for the physical field is (we use the same convention for indices with primes as in Sect. 2 , that is $1^{\prime}=3,3^{\prime}=1,2^{\prime}=2$ )

$$
\chi(\sigma)=\sum_{j} v_{j}(\varpi) \psi_{j j^{\prime}}(\sigma),
$$

where $v_{j}(\varpi)$ will be determined below. The braiding properties of the $\psi_{j k}$ fields follow from the ones of the $\psi$ and $\hat{\psi}$ fields, and from the fact that the braiding of a $\psi$ field with a $\hat{\psi}$ field is trivial:

$$
\psi_{j}(\sigma, \theta) \hat{\psi}_{k}\left(\sigma^{\prime}, \theta^{\prime}\right)=-\hat{\psi}_{k}\left(\sigma^{\prime}, \theta^{\prime}\right) \psi_{j}(\sigma, \theta) .
$$

One has

$$
\begin{aligned}
\psi_{j_{1} j_{2}}(\sigma, \theta) \psi_{k_{1} k_{2}}\left(\sigma^{\prime}, \theta^{\prime}\right)= & \sum_{l_{1}, l_{2}, m_{1}, m_{2}=1,2,3} S_{j_{1} k_{1}}^{l_{1} m_{1}}\left(\sigma-\sigma^{\prime}, \varpi\right) \hat{S}_{j_{2} k_{2}}^{l_{2} m_{2}}\left(\sigma-\sigma^{\prime}, \varpi+\varepsilon_{l_{1}}+\varepsilon_{l_{2}}\right) \\
& \times \psi_{l_{1} l_{2}}\left(\sigma^{\prime}, \theta^{\prime}\right) \psi_{m_{1} m_{2}}(\sigma, \theta) .
\end{aligned}
$$

Going back to the $\chi$-field, one considers the product $\chi(\sigma) \chi\left(\sigma^{\prime}\right)$, say for $\sigma>\sigma^{\prime}$, and applies (4.8) to each term coming from the expansion (4.6). Closure requires that the result be rewritten as a sum of products of operators of the type (4.6) (at this stage, one does not know how many physical operators are present). In any case, one has to check that, on the right-hand side, all terms that involve operators $\psi_{j k}$ with $k \neq j^{\prime}$ have vanishing coefficients. There are many conditions, and we shall only look at a typical one, since a complete proof will be obtained later on with a more sophisticated approach using $\xi$-fields. The vanishing of the coefficients of $\psi_{23}\left(\sigma^{\prime}\right) \psi_{12}(\sigma)$ and $\psi_{12}\left(\sigma^{\prime}\right) \psi_{23}(\sigma)$ give, respectively,

$$
\left\{\begin{array}{l}
v_{1}(\varpi) v_{2}(\varpi-(1-\pi / h)) \hat{S}_{32}^{32}(\varpi) S_{12}^{21}(\varpi)+v_{1}(\varpi) v_{2}(\varpi) \hat{S}_{23}^{32}(\varpi) S_{21}^{21}(\varpi)=0 \\
v_{1}(\varpi) v_{2}(\varpi-(1-\pi / h)) \hat{S}_{32}^{23}(\varpi) S_{12}^{12}(\varpi)+v_{1}(\varpi) v_{2}(\varpi) \hat{S}_{23}^{23}(\varpi) S_{21}^{12}(\varpi)=0 .
\end{array}\right.
$$

The determinant of this linear system must vanish. This is equivalent to the relation

$$
\frac{\sin ^{2} \hat{h}}{\sin ^{2} h}=\frac{\cos [\hat{h}(\hat{\omega}-1 / 2)] \cos [\hat{h}(\hat{\omega}+3 / 2)]}{\cos [h(\varpi+1 / 2)] \cos [h(\varpi-3 / 2)]} .
$$

Since $h+\hat{h}=0$, the ratio is one. The equation may be transformed into the condition

$$
0=\cos [h(2 \varpi-1)]-\cos [\hat{h}(2 \hat{\omega}+1)]=2 \sin \left[h \varpi+\hat{h} \hat{\omega}-\frac{h-\hat{h}}{2}\right] \sin [\hat{h} \hat{\omega}-h \varpi] .
$$

The condition of positivity for $\Delta_{0}(\varpi)$ is satisfied only by the vanishing of the second term, so that $\hat{h} \hat{\omega}-h \varpi=(\pi-h) \varpi$ must be a multiple of $\pi$. Since, it follows from (4.1) that $(\pi-h)(1-\pi / h)=2 \pi$, this is equivalent to the condition

$$
\varpi \equiv \varpi_{v}^{r}=\left(1-\frac{\pi}{h}\right)\left(\frac{r}{2}+v\right) \text {. }
$$

This determines the spectrum of highest weight. $r$ is an integer which should be restricted to the values 0 and 1 to avoid redundancy. We shall call $\mathscr{H}_{\text {phys }}$ the Hilbert 
space

$$
\mathscr{H}_{\mathrm{phys}} \equiv \bigoplus_{r=1,2} \bigoplus_{v} \mathscr{H}\left(\varpi_{v}^{r}\right)
$$

It only involves real positive highest weights, which correspond to unitary representations of the super-Virasoro algebra, and has a positive-definite metric. This is the Hilbert space where the exchange-algebra of the $\chi$-field will be shown to possess a consistent restriction.

One could go on to prove the truncation using $\psi$-fields, but the calculation is cumbersome and not very illuminating. We shall instead use the $\xi$-fields following the path of [3-5]. One proceeds in two steps. First one looks for linear combination of $\xi_{\alpha \alpha^{\prime}}$ that commute at different $\sigma$. For this, one relates the matrices $\varrho$ and $\hat{\varrho}$. One finds ${ }^{3}$

$$
\hat{\varrho}_{\alpha \beta}^{\gamma \delta}=\bar{\varrho}_{\gamma^{\prime} \delta^{\prime}}^{\alpha^{\prime} \boldsymbol{\beta}^{\prime}} i^{P(\alpha)+P(\beta)-P(\gamma)-P(\delta)}
$$

This relation is easily derived by explicit computations. It is understandable that, since $h$ and $\hat{h}$ are opposite, $\varrho$ is related to the inverse of $\varrho$, that is to $\varrho$. Next consider operators of the form

$$
\chi_{f}(\sigma) \equiv \sum f(\alpha) i^{P(\alpha)} \xi_{\alpha \alpha^{\prime}}
$$

It follows from Eqs. (4.14) and (3.11) that

$$
\chi_{f}(\sigma) \chi_{f}\left(\sigma^{\prime}\right)=\chi_{f}\left(\sigma^{\prime}\right) \chi_{f}(\sigma)
$$

if we assume that

$$
f(\alpha) f(\beta)=f(\gamma) f(\delta), \text { if } \varrho_{\alpha \beta}^{\gamma \delta} \neq 0 .
$$

The second step is to choose $f$ so that the physical Hilbert space (4.13) is left invariant. For this, $f$ should be such that it be possible to re-write the field $\chi_{f}$ in terms of $\psi_{j j}$-fields only. The appropriate choice is found by deriving an orthogonality-relation between $u$ and $\hat{u}$ similar to the one derived in [5] for the bosonic case. One starts from the relation (3.12), and deduces from the explicit expressions (3.4), (3.5) that

$$
\hat{u}_{\alpha}^{j}(\hat{\varpi})=e^{i(\hat{h} \hat{\omega}-h \varpi)[\alpha+j+1]}(-i)^{P(\alpha)} u_{\alpha}^{j}\left(\hat{\varpi}-\varepsilon_{j}\right) .
$$

In deriving this relation, one makes use of the fact that $\exp (2 i(\hat{h} \hat{\omega}-h \varpi))=1$ in agreement with (4.12). Combining the last equation with (3.12), one deduces

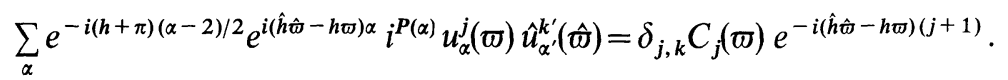

The last point is that we have to take account of the shifts of quasi-momenta. One may check that

$$
\hat{u}_{\alpha^{\prime}}^{k^{\prime}}\left(\hat{\varpi}+\varepsilon_{j} h / \pi\right)=(-1)^{\varepsilon_{j} \varepsilon_{k}} \hat{u}_{\alpha^{\prime}}^{k^{\prime}}(\hat{\varpi}),
$$

so that if we choose

$$
f(\alpha)=e^{-i(h+\pi)(\alpha-2) / 2} e^{i(\hat{h} \hat{w}-h \varpi) \alpha} i^{P(\alpha)},
$$

we find

$$
\chi=-\sum_{j} C_{j}(\varpi) e^{-i(\hat{h} \hat{\omega}-h \varpi)(j+1)} \psi_{j j^{\prime}}
$$

\footnotetext{
${ }^{3} i$ is always the root of -1 , not an index
} 
which leaves the physical space invariant. This completely determines the physical operator $\chi$ which we began to investigate above using the braiding of the $\psi$-fields. This operator is similar to the one which was first constructed $[16,3]$ for the bosonic case. Although the complete proof of the corresponding unitary truncation theorem remains to be given, it is likely that it holds: there probably exists a physical family of operators which, acting on $\mathscr{H}_{\text {phys }}$ are closed by fusion and braiding, and leave this unitary Hilbert space invariant. Thus supergravity should make sense for $C=5$, as was anticipated, in particular, in [18].

For the bosonic case, a more general result has been obtained [6,3-5], namely, it has been possible to treat the case where $h+\hat{h}=s \pi, s= \pm 1$ along a similar line as for $s=0$. Here the situation is more complicated if $s \neq 0$ as we now explain. Starting with the $\psi$ fields, one derives Eqs. $(4.9,4.10)$ again. The ratio is still one and Eq. (4.11) becomes

$$
2 \sin \left[h \varpi+\hat{h} \hat{\omega}-\frac{h-\hat{h}}{\pi}\right] \sin [\hat{h} \hat{\omega}-h \varpi+s \pi]=0 .
$$

This condition is realized if $\hat{h} \hat{\omega}-h \omega+s \pi$ is a multiple of $\pi$. However, in pursuing the calculation further, one finds many equations that are impossible to satisfy. From the viewpoint of the $\xi$ fields, one gets stuck as well, of course. There, the practical problem is that the matrix $\varrho$ involves $e^{i h / 2}$ which does not transform simply if $h$ is replaced by $\hat{h}=s \pi-h$. On the other hand, the striking properties of the super-Liouville string model in three dimensions seem to indicate that a unitary truncation theorem does hold at $C=7(s=1)$; it is not a straightforward extension of the case already worked out, however.

\section{Outlook}

We have determined the quantum-deformation of the super-Möbius group which underlies the super-Liouville theory. In the same way as in the bosonic case $[2,4$, 5], the quantum group structure will determine the fusion and braiding of the general chiral fields that appear in the chiral decomposition of powers of the metric. This will be instrumental in deriving the operator algebra of $N=1$ supergravity in two dimensions. For this purpose, there remains to study the $\xi$-fields belonging to higher representations, but this should be straightforward. A more tricky problem is to extend the discussion of Sect. 4, if possible, to the values $C=3$, and $C=7$ in the strong coupling regime, that are analogous to $C=7$, and $C=19$ of the bosonic case. The value $C=7$ is especially needed, since evidences have been given in [19], indicating that the corresponding non-critical string in three dimensions is related with the Ising model. On the other hand, our result for the case $C=5$ fully supports the validity of previous studies of the five-dimensional Liouville superstring [18], where the unitary truncation theorem was assumed to hold by analogy with the bosonic case. In particular, the relationship between the eigenvalue spectra of the quasi-momentum in the Ramond and Neveu-Schwarz sectors, which appeared in our discussion, is in agreement with the GSO projection which was used in [18] to construct the torus partition functions. The connection of the present point of view, and in particular of the special values of the central charge, with the occurrence of extended superconformal algebras in the corresponding world-sheet theories $[18,26,27]$ is worth further investigation. 
It is our hope that the quantum-group structure unravelled in the present paper will help in explicitly building the string-scattering amplitudes, thereby solving a long-standing problem. From the practical viewpoint, one may see that going from the Bloch-wave basis to the $\xi$-field basis leads to great simplifications. This is especially welcome because, as usual, the supersymmetric case is much more complex than the bosonic one.

From the viewpoint of quantum groups, it is of course interesting to study our structure per se. The simplest braiding matrix is nine-by-nine, and comparing our solution of Yang-Baxter equation with the analogous one previously associated with $\operatorname{osp}(1,2)$, we have seen that that there exist two solutions differing by signs in a few places, a fact which was unnoticed before to our knowledge. This illustrates once more the virtues of the present approach to quantum groups: since one explicitly constructs the operators whose braiding relations are given by the R-matrix, one may uncover unexpected novel solutions of Yang-Baxter's equation, and quantum-group structures. (This was also the case [7] for the Toda theories associated with Lie algebras of rank larger than 1.) Their common mathematical feature is that they ultimately follow from the monodromy properties of quantum operator-differential-equations. One may expect that there will be interesting developments in this direction, as well.

\section{Appendix A}

In this appendix, we recall some background-material contained in [8-10]. With our notation, the super-Liouville action reads

$$
S=\frac{1}{4 \pi \gamma} \int d \sigma d \tau\left[\frac{1}{2}\left(\partial_{a} \Phi\right)^{2}+\frac{i}{2} \bar{\Psi} \gamma^{a} \partial_{a} \Psi-\frac{1}{2} F^{2}+e^{\Phi} F-\frac{i}{2} \bar{\Psi} \Psi e^{\Phi}\right] .
$$

$\Phi$ is a bosonic field, and $\Psi$ is a Majorana spinor. Introducing the fermionic coordinates $\theta_{a}$ one defines the superfield

$$
\boldsymbol{\Phi}=\Phi+i \bar{\theta} \Psi+\frac{i}{2} \bar{\theta} \theta F .
$$

The general solution of the classical field equation may be written as follows [8]. Introduce the light-cone coordinates $u=\sigma+\tau, v=\sigma-\tau$ (we work with the Minkowski metric) and the standard covariant derivatives

$$
D_{1} \equiv-\frac{\partial}{\partial \theta_{2}}+2 i \theta_{2} \frac{\partial}{\partial u}, \quad D_{2} \equiv \frac{\partial}{\partial \theta_{1}}-2 i \theta_{1} \frac{\partial}{\partial v} .
$$

The field-equation may be written as

$$
D_{1} D_{2} \Phi+i e^{\mathbf{\Phi}}=0 .
$$

Superconformal transformations [28] are of the form [8],

$$
\begin{aligned}
& u^{\prime}=g_{1}\left(u, \theta_{2}\right), \quad \theta_{2}^{\prime}=\alpha_{1}\left(u, \theta_{2}\right) \text {, with } D_{1} g_{1}+2 i \alpha_{1} D_{1} \alpha_{1}=0 \text {, } \\
& v^{\prime}=g_{2}\left(v, \theta_{1}\right), \quad \theta_{1}^{\prime}=\alpha_{2}\left(v, \theta_{1}\right) \text {, with } D_{2} g_{2}+2 i \alpha_{2} D_{2} \alpha_{2}=0 \text {. }
\end{aligned}
$$


An easy computation leads to the chain rule

$$
D_{j}=-\eta_{j}\left(D_{j} \alpha_{j}\right) \Delta_{j}, \quad \Delta_{j} \equiv-\eta_{j}\left(\frac{\partial}{\partial \alpha_{j}}+2 i \alpha_{j} \frac{\partial}{\partial g_{j}}\right),
$$

where $\eta_{1}=-\eta_{2}=1$, so that the covariant derivatives are indeed covariant. The field-equation (A.4) is invariant under (A.5, A.6) if $\boldsymbol{\Phi}$ transforms in such a way that

$$
e^{\Phi} \rightarrow e^{\Phi}\left(-D_{1} \alpha_{1}\right)\left(D_{2} \alpha_{2}\right) \text {. }
$$

One may show [8] that the field-equation (A.4) is equivalent to two relations of the form

$$
\left[D_{1}^{3}-\mathscr{V}_{1}\left(u, \theta_{2}\right)\right] e^{-\Phi}=0, \quad\left[D_{2}^{3}-\mathscr{V}_{2}\left(v, \theta_{1}\right)\right] e^{-\Phi}=0 .
$$

The general solution of the field-equations (A.4) may thus be decomposed as

$$
e^{-\boldsymbol{\Phi}}=\sum_{j=1,3} f_{j}\left(u, \theta_{2}\right) \bar{f}_{j}\left(v, \theta_{1}\right)
$$

where the left (respectively right) chiral components $f_{j}$ (respectively $\bar{f}_{j}$ ) are any set of three independent solutions of the first (respectively the second) equation (A.9) which only depend upon one bosonic and one fermionic coordinates as indicated. Equation (A.10) is invariant under superconformal transformations, if, according to Eq. (A.8), the $f$ 's and $\bar{f}$ 's behave as

$$
f_{j} \rightarrow f_{j}\left(-D_{1} \alpha_{1}\right)^{-1}, \quad \bar{f}_{j} \rightarrow \bar{f}_{j}\left(D_{2} \alpha_{2}\right)^{-1}
$$

Since these two chiral components have similar properties at the present stage of the discussion, we consider only one of them, say $f_{j}$, and simply denote $\theta_{2}$ by $\theta, D_{1}$ by $D$, and $\mathscr{V}_{1}$ by $\mathscr{V}$ hereafter. As we have just recalled, the $f$ 's satisfy the supersymmetric Schrödinger equation

$$
\left[D^{3}-\mathscr{V}(u, \theta)\right] f_{j}=0 \text {. }
$$

A simple computation shows that $D^{3}=2 i \partial^{2} / \partial u \partial \theta+4 \theta \partial^{2} /(\partial u)^{2}$ so that if $\mathscr{V}=0$, the solutions are simply $f_{1}^{(0)}=1, f_{2}^{(0)}=\sqrt{2 i} \theta$, and $f_{3}^{(0)}=u$. Two are thus bosonic and one is fermionic. There is always a choice of independent solutions so that this is true in general. By convention, the lower index 2 is reserved for the fermionic solution. If we write the three solutions of (A.9) as column-vectors $f$ and $\bar{f}$, one sees that Eq. (A.10) is invariant under the transformations $f \rightarrow M f, \bar{f} \rightarrow\left(M^{T}\right)^{-1} \bar{f}$, where $M$ is a three-by-three matrix. This amounts to taking different solutions of (A.12). If one wants to preserve the commuting characters of the solutions, $M$ has five commuting and four anticommuting entries. An important subgroup of matrices $M$ is the one of super-Möbius transformations. It appears as follows. Consider the case where $\mathscr{V}=0$. In infinitesimal form, the subset of constant or linear superconformal transformations is generated by translations $(u \rightarrow u+\varepsilon, \theta \rightarrow \theta)$, dilatations $(u \rightarrow u-\varepsilon u, \theta \rightarrow \theta-\varepsilon \theta / 2)$, inversions $\left(u \rightarrow u-\varepsilon u^{2}, \theta \rightarrow \theta-\varepsilon \theta u\right)$, constant supersymmetries

$$
(u \rightarrow u+\sqrt{2 i} \eta \theta, \theta \rightarrow \theta+\eta / \sqrt{2 i}),
$$

linear supersymmetries

$$
(u \rightarrow u-\sqrt{2 i} \eta \theta u, \theta \rightarrow \theta-\eta u / \sqrt{2 i}) .
$$


The quantity $\varepsilon$ (respectively $\eta$ ) is an infinitesimal parameter that commutes (respectively anticommutes) with $\theta$. One easily verifies that, according to the transformation law (A.11), for $\mathscr{V}=0$, these super-Möbius transformations amount to taking linear combinations of the solutions $f_{j}^{(0)}$ of (A.4). Writing them as a column vector with $f_{3}^{(0)}, f_{2}^{(0)}$, and $f_{1}^{(0)}$ in downward order, one sees that the transformations may be written as

$$
\begin{gathered}
f^{(0)} \rightarrow\left(1+\varepsilon L_{1}\right) f^{(0)}, \quad f^{(0)} \rightarrow\left(1+\varepsilon L_{0}\right) f^{(0)}, \quad f^{(0)} \rightarrow\left(1+\varepsilon L_{-1}\right) f^{(0)}, \\
f^{(0)} \rightarrow\left(1+\eta G_{1 / 2}\right) f^{(0)}, \quad \text { and } \quad f^{(0)} \rightarrow\left(1+\eta G_{-1 / 2}\right) f^{(0)},
\end{gathered}
$$

respectively, where the action of the infinitesimal generators $\left(L_{0}, L_{ \pm 1}, G_{ \pm 1 / 2}\right)$ on the column vector $f^{(0)}$ is that associated with the fundamental representation of the super-Möbius algebra, i.e. that obtained by combining (3.15) and (3.17). For general $\mathscr{V}$ one may consider the same linear combinations of the $f_{j}$ 's, so that the super-Möbius group is indeed realized by particular choices of the above matrices $M$.

Since we are dealing with functions of $u$ only, we may equivalently work at fixed time $\tau$, say $\tau=0$, and replace $u$ by $\sigma$ everywhere as we have done in the main body of the paper. Thus we are working on the unit circle. If we write

$$
\mathscr{V}(\sigma, \theta)=G(\sigma)+\theta T(\sigma), \quad f(\sigma, \theta)=f^{0}(\sigma)+\theta f^{1}(\sigma)
$$

Eq. (A.12) becomes

$$
-4 f^{0^{\prime \prime}}+T f^{0}+G f^{1}=0, \quad-2 i f^{1^{\prime}}+G f^{0}=0,
$$

where primes denote derivatives with respect to $\sigma$. The boundary conditions are always such that $T$ and $G$ obey (anti)periodic conditions with period say $2 \pi$. One has

$$
T(\sigma+2 \pi)=T(\sigma), \quad G(\sigma+2 \pi)=\varsigma G(\sigma),
$$

where $\varsigma=1$ in the Ramond sector, and $\varsigma=-1$ in the Neveu-Schwarz sector. The last equation gives $\mathscr{V}(\sigma+2 \pi, \theta)=\mathscr{V}(\sigma, \varsigma \theta)$, and there exist three Bloch-wave solutions noted $\psi_{j}$ which are periodic up to a phase so that

$$
\begin{aligned}
& \psi_{1}(\sigma+2 \pi, \theta)=e^{-4 \pi i \gamma \varpi} \psi_{1}(\sigma, \varsigma \theta) \\
& \psi_{2}(\sigma+2 \pi, \theta)=\varsigma \psi_{2}(\sigma, \varsigma \theta) \\
& \psi_{3}(\sigma+2 \pi, \theta)=e^{4 \pi i \gamma \varpi} \psi_{3}(\sigma, \varsigma \theta)
\end{aligned}
$$

The quantity $\varpi$ so defined is related to the quasi-momentum of the Bloch waves. Next, free-field decomposition is achieved by writing $\psi_{j}(\sigma, \theta)=\psi_{j}^{0}(\sigma)+\theta \psi_{j}^{1}(\sigma)$, and letting

$$
\frac{\psi_{j}^{0^{\prime}}(\sigma)}{\psi_{j}^{0}(\sigma)} \equiv \sqrt{\gamma} p^{(j)}(\sigma), \quad \frac{\psi_{j}^{1}(\sigma)}{\psi_{j}^{0}(\sigma)} \equiv \sqrt{\gamma} b^{(j)}(\sigma),
$$

for $j=1$, and 3. The fields so defined are (anti)periodic:

$$
p^{(j)}(\sigma)=\sum_{n} e^{-i n \sigma} p_{n}^{(j)}, \quad b^{(j)}(\sigma)=\sum_{n} e^{-i n \sigma} b_{n}^{(j)},
$$


where the second sum runs over integer (half-integers) in the Ramond (NeveuSchwarz) sector. The modes satisfy the free-field Poisson-bracket relations, for fixed $j=1$, or 3 ,

$$
i\left\{p_{n}^{(j)}, p_{m}^{(j)}\right\}_{\text {P.в. }}=n \delta_{n,-m}, \quad i\left\{b_{n}^{(j)}, b_{m}^{(j)}\right\}_{\text {P.B. }}=\delta_{n,-m},
$$

as a consequence of the canonical Poisson-brackets associated with the action (A.1) [8]. The potentials $T$ and $G$ are the density of conformal and superconformal transformation generators respectively, and one may check that (A.18) does lead to the corresponding Poisson-bracket algebra with central charge $C=2 / \gamma$. The Poisson-brackets of $p_{n}^{(1)}$ with $p_{m}^{(3)}$ or of $b_{n}^{(1)}$ with $b_{m}^{(3)}$ are complicated and not very illuminating. The interesting structure comes out by looking at the Poissonbrackets of the $\psi$-fields. They form a closed algebra in the sense that one has

$$
\left\{\psi_{j}(\sigma, \theta), \psi_{k}\left(\sigma^{\prime}, \theta^{\prime}\right)\right\}_{\mathrm{B} . \mathrm{P} .}=\sum_{l, m} s_{j k}^{l m}\left(\sigma-\sigma^{\prime}, \varpi\right) \psi_{l}\left(\sigma^{\prime}, \theta^{\prime}\right) \psi_{m}(\sigma, \theta)
$$

Finally, quantization is achieved after replacing (A.19) by the corresponding commutators. There is a way to define the $\psi_{j}$ fields so that a quantum-version of (A.12) holds. For this purpose quantum-modifications must be introduced in the classical formulae recalled above. There are two possibilities so that the number of fields doubles. In particular there are fields $\psi_{j}^{0}$ and $\hat{\psi}_{j}^{0}$ with $j=1$, and 3 which are given by

$$
\psi_{j}^{0}=d_{j} N^{(j)}\left(e^{\sqrt{h / \pi} \phi_{j}}\right), \quad \hat{\psi}_{j}^{0}=\hat{d}_{j} N^{(j)}\left(e^{\sqrt{\hat{h} / \pi} \phi_{j}}\right), \quad j=1,3 .
$$

where $\phi_{j}^{\prime}=p^{(j)}$, and

$$
h=\frac{\pi}{4}(C-5-\sqrt{(C-9)(C-1)}), \quad \hat{h}=\frac{\pi}{4}(C-5+\sqrt{(C-9)(C-1)}) .
$$

The fields with quantum-modifications $h$ and $\hat{h}$ are noted $\psi_{j}$, and $\hat{\psi}_{j}$ respectively. For $C \rightarrow \infty, h \sim 2 \pi \gamma$, and the unhatted fields describe smooth modifications in the classical limit. The hatted fields, on the contrary are non-perturbative solutions. The quantum-version of the monodromy properties (A.16) is

$$
\begin{aligned}
& \psi_{1}(\sigma+2 \pi, \theta)=e^{i h} e^{-2 i h \varpi} \psi_{1}(\sigma, \varsigma \theta), \\
& \psi_{2}(\sigma+2 \pi, \theta)=\varsigma \psi_{2}(\sigma, \varsigma \theta), \\
& \psi_{3}(\sigma+2 \pi, \theta)=e^{i h} e^{2 i h \varpi} \psi_{3}(\sigma, \varsigma \theta) .
\end{aligned}
$$

with similar formulae for the hatted fields. From (A.21) it follows that $\varpi$ and $\hat{\varpi}$ are related to the zero-modes of the $\phi_{j}$-fields by

$$
\varpi=i p_{0}^{(1)} \sqrt{\frac{\pi}{h}} ; \quad \hat{\varpi}=i p_{0}^{(1)} \sqrt{\frac{\pi}{h}} ; \quad \hat{\varpi}=\varpi \frac{h}{\pi} ; \quad \varpi=\hat{\varpi} \frac{\hat{h}}{\pi} .
$$

The quantum version of the Poisson-bracket relations (A.20) is derived from the monodromy properties of the quantum-equivalent of (A.12). One has [10]

$$
\psi_{j}(\sigma, \theta) \psi_{k}\left(\sigma^{\prime}, \theta^{\prime}\right)=\sum_{l, m=1,2,3} S_{j k}^{l m}\left(\sigma-\sigma^{\prime}, \varpi\right) \psi_{l}\left(\sigma^{\prime}, \theta^{\prime}\right) \psi_{m}(\sigma, \theta)
$$


where ${ }^{4}$

$$
\begin{aligned}
& S_{13}^{13}(x, \varpi)=S_{31}^{31}(x,-\varpi)=\frac{\sin (h) \cos (h / 2)}{\cos [h(\varpi-1 / 2)] \sin [h \varpi]} e^{-i h(2 \varpi-1) \varepsilon} \\
& S_{13}^{31}(x, \varpi)=S_{31}^{13}(x,-\varpi)=-\frac{\lambda_{31} \sin [h(\varpi+1)]}{\lambda_{13} \sin [h \varpi]} e^{i h \varepsilon} \\
& S_{13}^{22}(x, \varpi)=S_{31}^{22}(x,-\varpi)=i \varepsilon \frac{\lambda_{22}}{\lambda_{13}} \frac{\sin (h)}{\cos [h(\varpi-1 / 2)]} e^{-i h(\varpi-1 / 2) \varepsilon} \\
& S_{22}^{13}(x, \varpi)=S_{22}^{31}(x,-\varpi)=-i \varepsilon \frac{\lambda_{13}}{\lambda_{22}} \frac{\sin (h) \sin [h(\varpi-1)]}{\sin (h \varpi) \cos [h(\varpi-1 / 2)]} e^{-i h(\varpi-1 / 2) \varepsilon}, \\
& S_{22}^{22}(x, \varpi)=-\left(1+\frac{\sin ^{2} h}{\cos [h(\varpi+1 / 2)] \cos [h(\varpi-1 / 2)]}\right)
\end{aligned}
$$

and

$$
\begin{aligned}
& S_{12}^{12}(x, \varpi)=S_{32}^{32}(x,-\varpi)=-i \varepsilon \frac{\sin (h)}{\cos [h(\varpi-1 / 2)]} e^{-i h(\varpi-1 / 2) \varepsilon}, \\
& S_{12}^{21}(x, \varpi)=S_{32}^{23}(x,-\varpi)=\frac{\lambda_{12}}{\lambda_{21}} \frac{\cos [h(\varpi+1 / 2)]}{\cos [h(\varpi-1 / 2)]}, \\
& S_{21}^{21}(x, \varpi)=S_{23}^{23}(x,-\varpi)=-i \varepsilon \frac{\sin (h)}{\cos [h(\varpi-1 / 2)]} e^{i h(\varpi-1 / 2) \varepsilon}, \\
& S_{21}^{12}(x, \varpi)=S_{23}^{32}(x,-\varpi)=\frac{\lambda_{21}}{\lambda_{12}} \frac{\cos [h(\varpi-3 / 2)]}{\cos [h(\varpi-1 / 2)]} .
\end{aligned}
$$

According to (A.24), the set of quasi-momenta may be equivalently described in terms of $\varpi$ and $\hat{\varpi}$. In terms of the former variable, the shift of $\varpi$ and $\hat{\psi}$ are such that, for any function $f$,

$$
\left\{\begin{array}{l}
\psi_{j}(\sigma, \theta) f(\varpi)=f\left(\varpi+\varepsilon_{j}\right) \psi_{j}(\sigma, \theta) \\
\hat{\psi}_{j}(\sigma, \theta) f(\varpi)=f\left(\varpi+\varepsilon_{j} \pi / h\right) \hat{\psi}_{j}(\sigma, \theta),
\end{array}\right.
$$

where

$$
\varepsilon_{3}=-\varepsilon_{1}=1, \text { and } \varepsilon_{2}=0 .
$$

Denote by $\mathscr{H}(\varpi)$ the Fock space of states created by powers of the operators $p_{n}^{(1)}$, and $b_{m}^{(1)}$ (or equivalently of the operators $p_{n}^{(3)}$, and $b_{m}^{(3)}$ ), with $m$ and $n$ negative, acting on the highest-weight state $|\varpi, 0\rangle\rangle$. It is the eigenstate of $\varpi$ which is annihilated by the $p_{n}^{(1)}$, and $b_{m}^{(1)}$ (or equivalently by the operators $p_{n}^{(3)}$, and $b_{m}^{(3)}$ ) with $m$ and $n$ positive. The corresponding highest weights $\Delta_{0}(\varpi)$ such that $L_{0}|\varpi, 0\rangle$ $=\Delta_{0}(\varpi)|\varpi, 0\rangle$ are given by

$$
\Delta_{0}(\varpi)=\frac{C-1}{16}\left(\frac{1-\varsigma}{2}\right)-\frac{h}{2 \pi} \varpi^{2} .
$$

\footnotetext{
${ }^{4}$ Our notations are such that $2 h \omega$ and $h$ are equal to $\eta \omega$ and $\pi \hbar \eta^{2}$ of [10] respectively

5 This special notation for the kets is used to avoid possible confusion with the group-theoretic states $|J, M\rangle$ used in Sect. 3
} 
It follows from (A.28, A.29) that the fields $\psi$ and $\hat{\psi}$ live in spaces of the form

$$
\mathscr{H}_{\varpi^{(0)}}=\bigoplus_{v, \hat{v}=-\infty}^{\infty} \mathscr{H}\left(\varpi^{(0)}+v+\hat{v} \frac{\pi}{h}\right)
$$

where $v$ and $\hat{v}$ are integers, and $\varpi^{(0)}$ is an arbitrary constant. The spectrum of highest weights is given by

$$
\Delta_{0}\left(\varpi^{(0)}+v+\hat{v} \frac{\pi}{h}\right)=\frac{C-1}{16}\left(\frac{1-\varsigma}{2}\right)-\frac{1}{2}\left\{\left(\varpi^{(0)}+v\right) \sqrt{h / \pi}+\hat{v} \sqrt{h / \pi}\right\}^{2} .
$$

According to (A.22), Kac's formula reads, with our notations

$$
\Delta_{\mathrm{Kac}}(m, n) \equiv \frac{C-1}{16}\left(\frac{1-\varsigma}{2}\right)-\frac{1}{8}\{m \sqrt{h / \pi}+n \sqrt{h / \pi}\}^{2},
$$

where $m$ and $n$ are positive integers. Comparing with (A.32) one sees that the two expressions coincide if we let

$$
\varpi^{(0)}=\varpi^{(00)} \equiv \frac{1}{2}(1+\pi / h)
$$

and $2 v=n-1,2 \hat{v}=m-1 . \varpi^{(00)}$ is the quasi-momentum of the $S l(2, C)$-invariant vacuum which is such that $\Delta_{0}\left(\varpi^{(00)}\right)=0$ in the Neveu-Schwarz sector. In the Ramond sector, $\varpi^{(00)}=0$ is the analogous value, giving $\Delta_{0}\left(\varpi^{(00)}\right)=0$ as well; more generally, it seems natural that the Hilbert space associated with the Ramond sector should be as in (A.31), with $v$ and $\hat{v}$ half-integers instead of integers. This correspondence is related to the shift of quasi-momentum occurring in the comparison of the exchange matrix for the Bloch waves in the two sectors. This seems to imply that, in a future formulation of the theory, the spin fields $\mathscr{S}$ (respectively $\widehat{\mathscr{S}}$ ) - whose insertions create endpoints of two-sheeted branch cuts for $\psi$ (respectively $\hat{\psi}$ ) - should also shift $\varpi$ by $\pi / 2 h$ (respectively $\pi / 2 \hat{h}$ ). Different choices of $\varpi^{(0)}$ are introduced in addition, for various reasons, in particular in the strong coupling regime [see Eq. (4.12)].

\section{Appendix B}

In this appendix we give a more detailed description of the change of basis from the $\psi$ fields which appear naturally in the operator algebra of two-dimensional supergravity to the $\xi$ fields which exhibit its relation to the quantum super-Möbius group. The first step consists in choosing a more convenient normalization of the $\psi$ fields, in order to simplify the exchange matrix of Eq. (A.26, A.27) and in particular to eliminate its dependence on the quantities $\lambda_{j k}$. In other words we make a different choice of the functions $d_{j}$ appearing in Eq. (A.21). Indeed let us set

$$
\tilde{d}_{j}(\varpi)=\mu_{j}(\varpi) d_{j}(\varpi) ; \quad j=1,2,3 .
$$

Under such a change of normalizations the braiding matrix transforms via

$$
\tilde{S}_{j k}^{l m}(x, \varpi)=\frac{\mu_{j}(\varpi) \mu_{k}\left(\varpi+\varepsilon_{j}\right)}{\mu_{l}(\varpi) \mu_{m}\left(\varpi+\varepsilon_{l}\right)} S_{j k}^{l m}(x, \varpi) .
$$


A convenient choice is

$$
\left\{\begin{array}{l}
\mu_{1}(\varpi)=\frac{\lambda_{31}(\varpi-1)}{\cos [h(\varpi-1 / 2)]} \\
\mu_{2}(\varpi)=\frac{1}{\lambda_{21}(\varpi) \cos [h(\varpi-1 / 2)]} \\
\mu_{3}(\varpi)=\frac{1}{\sin (h \varpi)}
\end{array}\right.
$$

In order to show that with this new normalization the quantities $\lambda_{j k}$ indeed cancel from the braiding matrix, it is necessary to use the following identities, valid for some choice of the normalizations of [10]

$$
\begin{gathered}
\lambda_{31}(-\varpi)=\lambda_{13}(\varpi) ; \quad \frac{\lambda_{13}(\varpi)}{\lambda_{31}(\varpi-1)}=-\frac{\sin [h \varpi]}{\sin [h(\varpi-1)]}, \\
\lambda_{12}(\varpi)=\lambda_{21}(-\varpi+1) ; \quad \frac{\lambda_{12}(\varpi)}{\lambda_{12}(-\varpi)}=\frac{\cos [h(\varpi+1 / 2)]}{\cos [h(\varpi-1 / 2)]}, \\
\lambda_{22}(\varpi)=\lambda_{22}(-\varpi) ; \quad \lambda_{22}(\varpi)\left(\lambda_{21}(\varpi)\right)^{2}=\frac{\cos [h(\varpi+1 / 2)]}{2 \sin (h / 2) \cos [h(\varpi-1 / 2)]} .
\end{gathered}
$$

The identities of the first line of Eq. (B.4), as well as the first one of the third line are explicitly stated in [10] [below Eq. (5.11)]; those on the second line can be derived from the expressions ${ }^{6}$ for $\lambda_{12}$ and $\lambda_{21}$ given after Eq. (6.8) of [10]. Finally the second identity on the third line can be derived from the expressions for $\lambda_{22}$ [below Eq. (5.11)] and $\lambda_{21}$. Regarding this last identity it may seem at first sight surprising that the rational factor in the expression for $\lambda_{22}$, in which $\varpi$ appears without its usual factor $h$, may be eliminated, however it turns out that the ratios of gamma functions which appear are precisely taylored to cancel it (to prove that, one must make use of the second degree equation which $h$ satisfies). To summarize, the identities (B.4) imply that, with the normalizations of Eq. (B.3), the braiding algebra of the $\psi$ fields becomes, as quoted in Sect. 2 (we omit the tildes)

$$
\begin{aligned}
& S_{13}^{13}(x, \varpi)=S_{31}^{31}(x,-\varpi)=\frac{\sin (h) \cos (h / 2)}{\cos [h(\varpi-1 / 2)] \sin [h \varpi]} e^{-i h(2 \varpi-1) \varepsilon}, \\
& S_{13}^{31}(x, \varpi)=S_{31}^{13}(x,-\varpi)=\frac{\sin [h(\varpi+1)] \cos [h(\varpi+1 / 2)]}{\sin [h \varpi] \cos [h(\varpi-1 / 2)]} e^{i h \varepsilon}, \\
& S_{13}^{22}(x, \varpi)=S_{31}^{22}(x,-\varpi)=i \varepsilon \frac{\cos (h / 2) \cos [h(\varpi+1 / 2)]}{\sin [h \varpi] \cos [h(\varpi-1 / 2)]} e^{-i h(\varpi-1 / 2) \varepsilon}, \\
& S_{22}^{13}(x, \varpi)=S_{22}^{31}(x,-\varpi)=-i \varepsilon \frac{2 \sin (h / 2) \sin (h) \sin [h(\varpi-1)]}{\cos [h(\varpi-1 / 2)] \cos [h(\varpi+1 / 2)]} e^{-i h(\varpi-1 / 2) \varepsilon}, \\
& S_{22}^{22}(x, \varpi)=-\left(1+\frac{\sin ^{2} h}{\cos [h(\varpi+1 / 2)] \cos [h(\varpi-1 / 2)]}\right),
\end{aligned}
$$

\footnotetext{
6 Warning: these two expressions contain a misprint, the numerators and denominators of the fraction appearing on the right-hand side should be exchanged
} 
and

$$
\begin{aligned}
& S_{12}^{12}(x, \varpi)=S_{32}^{32}(x,-\varpi)=-i \varepsilon \frac{\sin (h)}{\cos [h(\varpi-1 / 2)]} e^{-i h(\varpi-1 / 2) \varepsilon}, \\
& S_{12}^{21}(x, \varpi)=S_{32}^{23}(x,-\varpi)=\frac{\cos [h(\varpi+1 / 2)]}{\cos [h(\varpi-1 / 2)]} \\
& S_{21}^{21}(x, \varpi)=S_{23}^{23}(x,-\varpi)=-i \varepsilon \frac{\sin (h)}{\cos [h(\varpi-1 / 2)]} e^{i h(\varpi-1 / 2) \varepsilon} \\
& S_{21}^{12}(x, \varpi)=S_{23}^{32}(x,-\varpi)=\frac{\cos [h(\varpi-3 / 2)]}{\cos [h(\varpi-1 / 2)]}
\end{aligned}
$$

As announced the quantities $\lambda_{j k}$ have been eliminated, moreover in the new normalization the exchange matrix has a number of useful properties, on which relies the construction of the new basis of fields. Indeed it follows from Eq. (B.5, B.6) that

$$
\sum_{l, m} S_{l m}^{j k}(x, \varpi)=e^{-i h e} \text { for all values of }(j, k) .
$$

Following previous studies of similar problems $[2,7]$ we recall that such identities signal the possibility of constructing combinations of the $\psi$ fields whose braiding will be especially simple. Namely if we consider

$$
\xi_{\alpha}(\sigma, \theta)=e^{-Y_{\alpha}(\varpi)}\left(\sum_{j} \psi_{j}(\sigma, \theta) e^{C_{\alpha}^{j}}\right) e^{Y_{\alpha}(\varpi)},
$$

then the braiding of $\xi_{\alpha}$ with itself involves precisely the quantities appearing in Eq. (B.7); indeed

so that Eq. (B.7) implies

$$
\xi_{\alpha}(\sigma, \theta) \xi_{\alpha}\left(\sigma^{\prime}, \theta^{\prime}\right)=e^{-Y_{\alpha}}\left(\sum_{j, k} e^{C_{\alpha}^{j}+C_{\alpha}^{k}} S_{j k}^{l m}(x, \varpi) \psi_{l}\left(\sigma^{\prime}, \theta^{\prime}\right) \psi_{k}(\sigma, \theta)\right) e^{Y_{\alpha}}
$$

$$
\xi_{\alpha}(\sigma, \theta) \xi_{\alpha}\left(\sigma^{\prime}, \theta^{\prime}\right)=\varrho_{\alpha \alpha}^{\alpha \alpha} \xi_{\alpha}\left(\sigma^{\prime}, \theta^{\prime}\right) \xi_{\alpha}(\sigma, \theta) ; \quad \varrho_{\alpha \alpha}^{\alpha \alpha}=e^{-i h \varepsilon},
$$

provided the quantities $C_{\alpha}^{j}$ are such that

$$
C_{\alpha}^{j}+C_{\alpha}^{k}=C_{\alpha}^{l}+C_{\alpha}^{m} \quad \text { whenever } \quad S_{j k}^{l m}(x, \varpi) \neq 0 .
$$

In order to study the braiding of $\xi_{\alpha}$ and $\xi_{\beta}$ for $\alpha \neq \beta$ we will have to derive a number of other identities satisfied by the braiding matrix of Eqs. (B.5, B.6). Indeed, when studying the exchange of $\xi_{\alpha}$ and $\xi_{\beta}$, it is convenient to separate the terms involving $Y_{\alpha} \pm Y_{\beta}$ by writing

$$
\begin{aligned}
& \xi_{\alpha}(\sigma, \theta) \xi_{\beta}\left(\sigma^{\prime}, \theta^{\prime}\right)=e^{-\left(Y_{\alpha}+Y_{\beta}\right) / 2}\left[e^{-\left(Y_{\alpha}-Y_{\beta}\right) / 2}\left(\sum_{j} \psi_{j}(\sigma, \theta) e^{C_{\alpha}^{j}}\right) e^{\left(Y_{\alpha}-Y_{\beta}\right) / 2}\right] \\
& \times\left[e^{\left(Y_{\alpha}-Y_{\beta}\right) / 2}\left(\sum_{k} \psi_{k}\left(\sigma^{\prime}, \theta^{\prime}\right) e^{C_{\alpha}^{k}}\right) e^{-\left(Y_{\alpha}-Y_{\beta}\right) / 2}\right] e^{\left(Y_{\alpha}+Y_{\beta}\right) / 2} .
\end{aligned}
$$

As a first step, we would like to derive an exchange equation of the form:

$$
\begin{aligned}
& {\left[e^{-\left(Y_{\alpha}-Y_{\beta}\right) / 2}\left(\sum_{j} \psi_{j}(\sigma, \theta) e^{C_{\alpha}^{j}}\right) e^{\left(Y_{\alpha}-Y_{\beta}\right) / 2}\right] \times\left[e^{\left(Y_{\alpha}-Y_{\beta}\right) / 2}\left(\sum_{k} \psi_{k}\left(\sigma^{\prime}, \theta^{\prime}\right) e^{C_{\alpha}^{k}}\right) e^{-\left(Y_{\alpha}-Y_{\beta}\right) / 2}\right]} \\
& =\sum_{\gamma, \delta} \varrho_{\alpha \beta}^{\gamma \delta}\left[e^{-\left(Y_{\gamma}-Y_{\delta}\right) / 2}\left(\sum_{j} \psi_{j}\left(\sigma^{\prime}, \theta^{\prime}\right) e^{C_{\gamma}^{j}}\right) e^{\left(Y_{\gamma}-Y_{\delta}\right) / 2}\right] \\
& \quad \times\left[e^{\left(Y_{\gamma}-Y_{\delta}\right) / 2}\left(\sum_{k} \psi_{k}(\sigma, \theta) e^{C_{\delta}^{k}}\right) e^{-\left(Y_{\gamma}-Y_{\delta}\right) / 2}\right]
\end{aligned}
$$


which only involves the differences $Y_{\gamma}-Y_{\delta}$. The sums $Y_{\gamma}+Y_{\delta}$ are determined as follows: Looking at Eq. (B.12) with $\alpha$ and $\beta$ replaced by $\gamma$ and $\delta$ respectively, one sees that Eq. (B.13) will take the form of the exchange algebra (3.2) for the fields $\xi$ if the terms which appear in the summation are such that

$$
Y_{\alpha}+Y_{\beta}=Y_{\gamma}+Y_{\delta} \text {, }
$$

(up to an irrelevant constant $c_{\alpha \beta \gamma \delta}$ ) so that $e^{ \pm\left(Y_{\gamma}+Y_{\delta}\right) / 2}$ are common factors and may be freely multiplied on the right and on the left. To proceed further we consider the ansatz:

$$
Y_{\alpha}=\varpi^{2} i v_{\alpha}+\varpi D_{\alpha}
$$

and set

$$
\varphi_{\alpha \beta}^{j k} \equiv C_{\alpha}^{j}+C_{\beta}^{k}+\frac{1}{2}\left(D_{\alpha}-D_{\beta}\right)\left(\varepsilon_{j}-\varepsilon_{k}\right)+i \frac{h}{2}\left(\varepsilon_{j}^{2}-\varepsilon_{k}^{2}\right)+i h v_{\alpha \beta} \varepsilon_{j} \varepsilon_{k},
$$

where $v_{\alpha \beta}=v_{\alpha}-v_{\beta}$. Substituting (B.15) into (B.13), we find an equation of the form

$$
\begin{array}{r}
\sum_{j k, l m}\left[S_{j k}^{l m}(x, \varpi) \exp \left(i h v_{\alpha \beta} \varpi\left(\varepsilon_{j}-\varepsilon_{k}\right)+\varphi_{\alpha \beta}^{j k}\right) \psi_{l}\left(\sigma^{\prime}, \theta^{\prime}\right) \psi_{m}(\sigma, \theta)\right] \\
=\sum_{\gamma, \delta} \varrho_{\alpha \beta}^{\gamma \delta} \sum_{l m}\left[\exp \left(\varphi_{\gamma \delta}^{l m}+i h v_{\gamma \delta} \varpi\left(\varepsilon_{l}-\varepsilon_{m}\right)\right) \psi_{l}\left(\sigma^{\prime}, \theta^{\prime}\right) \psi_{m}(\sigma, \theta)\right] .
\end{array}
$$

For any value of $(l, m)$, the coefficient of $\psi_{l}\left(\sigma^{\prime}, \theta^{\prime}\right) \psi_{m}(\sigma, \theta)$ on the right-hand side of Eq. (B.17) has a finite number of Fourier components with respect to $\varpi$. This shows that an equality such as (B.17) is rather non-trivial since most of the $S$-matrix elements appearing on the left-hand side have poles for some values of $\varpi$, thus the essential idea behind our construction is that it is possible to define combinations of these matrix elements for which these poles cancel, and moreover which have only a finite number of Fourier components with respect to $\varpi$. Fourier analysis shows that $v_{\gamma \delta}$ is an integer. No $v_{\gamma \delta}$ may vanish, since otherwise the determinant of $u_{\alpha}^{j}$ would be zero for any $\varpi$. Thus all $v_{\alpha}$ 's must be different. We now consider separately the quantities appearing on the left-hand side of (B.17) as coefficients of $\psi_{l}\left(\sigma^{\prime}, \theta^{\prime}\right) \psi_{m}(\sigma, \theta)$ for the various values of $(l, m)$.

The case $l=2, m=1$. The quantity

$$
T_{\alpha \beta}^{21}\left(v_{\alpha \beta}\right) \equiv e^{i h v_{\alpha \beta}+\varphi_{\alpha \beta}^{21}} S_{21}^{21}+e^{-i h v_{\alpha \beta}+\varphi_{\alpha \beta}^{12}} S_{12}^{21}
$$

must have a finite number of Fourier coefficients, in particular the poles in $z=e^{i h \pi}$ must disappear. This is true if

$$
\varphi_{\alpha \beta}^{12}-\varphi_{\alpha \beta}^{21}=i v_{\alpha \beta}(h+\pi) .
$$

Assuming this condition to hold, one finds by explicit computation

$$
\begin{aligned}
T_{\alpha \beta}^{21}(v)= & e^{\left(\varphi^{12}+\varphi^{21}\right) / 2} e^{-i h}\left\{\frac{\left(1-e^{2 i h}\right)}{1+z^{2} e^{-i h}}\left[\left(-i z e^{-i h / 2}\right)^{-v}-\left(-i z e^{-i h / 2}\right)^{2+\nu} \cdot\right]\right. \\
& \left.+e^{2 i h}\left(-i z e^{-i h / 2}\right)^{-v}\right\},
\end{aligned}
$$

where the terms are arranged so that the Fourier decomposition is immediate. 
The case $l=1, m=3$. Consider

$$
T_{\alpha \beta}^{13}\left(v_{\alpha \beta}\right) \equiv e^{2 i h v_{\alpha \beta}+\varphi_{\alpha \beta}^{31}} S_{31}^{13}+e^{-2 i h v_{\alpha \beta}+\varphi_{\alpha \beta}^{13}} S_{13}^{13}+e^{\varphi_{\alpha \beta}^{22}} S_{22}^{13} .
$$

The poles in $x=e^{2 i h m}$ disappear if

$$
\varphi_{\alpha \beta}^{31}=\varphi_{\alpha \beta}^{13} ; \quad \varphi_{\alpha \beta}^{31}=\varphi_{\alpha \beta}^{22}+i v_{\alpha \beta}(h+\pi) .
$$

One finds

$$
\begin{aligned}
& e^{-\varphi_{\alpha \beta}^{31}} T_{\alpha \beta}^{13}(v)=e^{3 i h}\left\{e^{-i h}\left(1-e^{-2 i h}\right) \frac{x^{-v}-x^{v}}{1-x}\right. \\
& \left.-e^{-2 i h}\left(1-2 e^{-i h}\right) x^{\nu}-\frac{e^{-3 i h}}{1+e^{i h}} x^{\nu}(1-x)\right\} \\
& +e^{3 i h}(-1)^{v+1} e^{i v h} \frac{\left(1-e^{-2 i h}\right)^{2}}{1+e^{-i h}} \\
& \times\left\{\frac{1+e^{-i h}}{1-e^{2 i h}} \frac{1-\left(-x e^{-i h}\right)^{-v}}{1-\left(-x e^{-i h}\right)}-\frac{e^{-i h}}{1-e^{2 i h}}\right\} \\
& +e^{3 i h}(-1)^{v+1} e^{i h} \frac{1-x e^{-2 i h}}{1+e^{-i h}} \\
& \left.\times\left\{\left(1-e^{-2 i h}\right) \frac{1-\left(-x e^{i h}\right)^{v}}{1-\left(-x e^{i h}\right)}-\left(-x e^{i h}\right)^{v} e^{-2 i h}\right)\right\} .
\end{aligned}
$$

The case $l=2, m=2$. Consider

$$
T_{\alpha \beta}^{22}\left(v_{\alpha \beta}\right) \equiv e^{2 i h v_{\alpha \beta}+\varphi_{\alpha \beta}^{31}} S_{31}^{22}+e^{-2 i h v_{\alpha \beta}+\varphi_{\alpha \beta}^{13}} S_{13}^{22}+e^{\varphi_{\alpha \beta}^{22}} S_{22}^{22} .
$$

The poles in $x$ disappear under the same condition as above and one finds:

$$
\begin{gathered}
e^{-\varphi_{\alpha \beta}^{31}} T_{\alpha \beta}^{22}(v)=-1+\left\{x^{-v}\left(1+x e^{i h}\right)-x^{v+1}\left(x+e^{i h}\right)\right\} \frac{1}{1-x} \\
+(-1)^{v+1} e^{-i h(v+1)} \frac{1-\left(-x e^{-i h}\right)^{-v}}{1-\left(-x e^{-i h}\right)}+(-1)^{v} e^{i h(-v+1)} \frac{1-\left(-x e^{-i h}\right)^{-v+1}}{1-\left(-x e^{-i h}\right)} \\
+(-1)^{v+1} e^{i h(-v+1)} \frac{1-\left(-x e^{i h}\right)^{v+1}}{1-\left(-x e^{i h}\right)}+(-1)^{v} e^{-i h(v+1)} \frac{1-\left(-x e^{i h}\right)^{v+2}}{1-\left(-x e^{i h}\right)}
\end{gathered}
$$

The above formulae, with $v=0$ give:

$$
\begin{aligned}
& T_{\alpha \alpha}^{21}(0)=e^{\varphi_{\alpha \alpha}^{21}} e^{-i h}, \\
& T_{\alpha \alpha}^{13}(0)=e^{\varphi_{\alpha \alpha}^{13}} e^{-i h}, \\
& T_{\alpha \alpha}^{22}(0)=e^{\varphi_{\alpha \alpha}^{22}} e^{-i h},
\end{aligned}
$$

so that we recover the formulae we have used to derive the braiding of $\xi_{\alpha}$ with itself.

A closer consideration of the case $l=m=2$ shows that the method of $[2,7]$ cannot give a complete answer to our problem. Indeed the $l=m=2$ term on the right-hand side of Eq. (B.17) is obviously independent of $\varpi$, whereas the quantity $T_{\alpha \beta}^{22}(v)$ of Eq. (B.25) is $\varpi$ independent for $v=0$ and 1 only, so that we cannot define all three $\xi$ fields of the form of Eq. (B.8). It turns out that the solution is to define 
two of the fields (say $\xi_{1,3}$ ) of the form (B.8), with

$$
v_{1}=-v_{3}=\frac{1}{2} ; \quad D_{1}=D_{3}=0 ; \quad C_{1}^{1}=-C_{1}^{3}=i \pi ; \quad C_{1}^{2}=C_{3}^{1}=C_{3}^{2}=C_{3}^{3}=0 .
$$

From these values, which satisfy the constraints (B.19, B.22, B.11), and Eqs. (B.8, B.15) one easily recovers the quantities $u_{1,3}^{j}$ of Eq. (3.4). Moreover one may use the identities given above for $v= \pm 1$ to derive the braiding of $\xi_{1}$ with $\xi_{3}$. In the case $v=1$ one finds that the result can be cast in the form of Eq. (B.17), with $\varrho_{13}^{31}$ as the only non-vanishing matrix element appearing on the right-hand side. On the contrary, in the case $v=-1$, one finds that the third field $\xi_{2}$ inevitably appears on the right-hand side, which turns out to contain three terms, proportional to $\varrho_{31}^{13}$, $\varrho_{31}^{22}$, and $\varrho_{31}^{31}$, respectively. This computation fixes $\xi_{2}$ up to a normalisation constant, and reduces to (3.5) for a convenient choice of this constant. One may then use Yang-Baxter's equation to determine the other non-vanishing elements of the matrix $\varrho$, and their values, up to some minor indetermination. Finally an explicit computation lifts this indetermination and shows that the previously determined $\xi$ 's indeed satisfy the exchange algebra of Eq. (3.7). This computation makes use of the identities we have described above, and of others, most of which can be expressed as combinations of the former, but are in some cases simpler to derive directly from the explicit expression of the $S$-matrix; for instance:

$$
\cos (h \varpi)\left(S_{22}^{22}(x, \varpi)+1\right)-\frac{1}{2}\left(e^{i h \varpi} S_{13}^{22}(x, \varpi)+e^{-i h \varpi} S_{31}^{22}(x, \varpi)\right)=0 .
$$

Acknowledgements. We wish to thank O. Babelon for kindly explaining details of [10] and providing some related unpublished results. Part of this work was done while one of us (J.-L. G.) was visiting the Institute for Theoretical Physics at Santa Barbara. He benefited a great deal from the stimulating surrounding of the 1990 Workshop on Conformal Field Theory (in particular from discussions with H. Saleur); and is grateful for the warm hospitality and generous financial support extended to him. This research was supported in part by the National Science Foundation under Grant No. PHY89-04035, supplemented by funds from the National Aeronautics and Space Administration, at the University of California at Santa Barbara. The other (B.R.) is financially supported by the "Délégation Générale pour l'Armement" in the framework of the "Option Recherche."

\section{References}

1. Babelon, O.: Phys. Lett. B 215, 523 (1988)

2. Gervais, J.-L.: Commun. Math. Phys. 130, 257 (1990)

3. Gervais, J.-L., Rostand, B.: Nucl. Phys. B 346, 473 (1990)

4. Gervais, J.-L.: Phys. Lett. B 243, 85 (1990)

5. Gervais, J.-L.: Commun. Math. Phys. 138, 301 (1991)

6. Gervais, J.-L., Neveu, A.: Phys. Lett. 151B, 271 (1985)

7. Cremmer, E., Gervais, J.-L.: Commun. Math. Phys. 134, 619 (1990)

8. Arvis, J.F.: Nucl. Phys. B 212, 151 (1983)

9. Arvis, J.F.: Nucl. Phys. B 218, 309 (1983)

10. Babelon, O.: Nucl. Phys. B 258, 680 (1985)

11. Polyakov, A.M.: Phys. Lett. 103B, 207 (1981)

12. Gervais, J.-L., Neveu, A.: Nucl. Phys. B 199, 59 (1982)

13. Gervais, J.-L., Neveu, A.: Nucl. Phys. B 202, 125 (1982)

14. Gervais, J.-L., Neveu, A.: Nucl. Phys. B 224, 329 (1983)

15. Gervais, J.-L., Neveu, A.: Nucl. Phys. B 238, 125, 396 (1984)

16. Gervais, J.-L., Neveu, A.: Nucl. Phys. B 257 [FS14], 59 (1985) 
17. Gervais, J.-L., Neveu, A.: Nucl. Phys. B 264, 557 (1986)

18. Bilal, A., Gervais, J.-L.: Nucl. Phys. B 284, 397 (1987); Phys. Lett. B 187, 39 (1987); Nucl. Phys. B 293, 1 (1987); for reviews see [20]

19. Bilal, A., Gervais, J.-L.: Nucl. Phys. B 295 [FS21], 277 (1988)

20. For reviews see Gervais, J.-L.: Liouville superstrings. In: Perspectives in String, The Proc. of the Niels Bohr/Nordita Meeting, (1987), Singapore: World Scientific; DST workshop on particle physics-Superstring theory, Proc. of the I.I.T. Kanpur meeting (1987) Singapore: World Scientific; Gervais, J.-L.: Systematic approach to conformal theories. Nucl. Phys. B (Proc. Supp.) 5B, 119-136 (1988); Bilal, A., Gervais, J.-L.: Conformal theories with nonlinearly-extended Virasoro symmetries and Lie-algebras classification. Conference Proceedings: Infinite dimensional Lie algebras and Lie groups. V. Kac (ed.) Marseille 1988. Singapore: World Scientific

21. Kulish, P.: Quantum superalgebra osp $(1,2)$. Preprint 1988

22. Saleur, H.: Nucl. Phys. B 336, 363 (1990)

23. Gervais, J.-L.: On the algebraic structure of quantum gravity in two dimensions, ICTP preprint Trieste Conference on topological Methods in quantum field theories. Singapore: World Scientific

24. Kulish, P., Sklyanin, N.: J. Sov. Math. 19, $1596(1982)$

25. Kulish, P., Reshetikhin, N.: Lett. Math. Phys. 18, 143 (1989)

26. Kutasov, D., Seiberg, N.: Non-critical superstrings. Preprint PUPT-1193, RU-90-41

27. Callan, Jr., C.G., Harvey, J.A., Strominger, A.: World-sheet approach to heterotic instantons and solitons. Preprint EFI-91-03, PUPT-1233, UCSB-TH-91-74

28. Gervais, J.-L., Sakita, B.: Nucl. Phys. B 34, 832 (1971)

Communicated by K. Gawedzki 
